CFTP/09-021

\title{
CP-sensitive spin-spin correlations in neutralino production at the ILC
}

\author{
A. Bartl ${ }^{1}$, K. Hohenwarter-Sodek ${ }^{1}$, T. Kernreiter ${ }^{2}$, O. Kittel ${ }^{3}$ and M. Terwort ${ }^{4}$ \\ 1 Faculty of Physics, Universität Wien, \\ A-1090 Vienna, Austria \\ 2 Departamento de Física and CFTP, \\ Instituto Superior Técnico Av. Rovisco Pais 1, \\ 1049-001 Lisboa, Portugal \\ 3 Departamento de Física Teórica y del Cosmos and CAFPE, \\ Universidad de Granada, E-18071 Granada, Spain \\ 4 Institut für Experimentalphysik, Universität Hamburg \\ Notkestraße 85, D-22607 Hamburg, Germany
}

\begin{abstract}
We study the CP-violating terms of the spin-spin correlations in neutralino production and their subsequent two-body decays into sleptons plus leptons at the ILC. We analyze CP-sensitive observables with the help of T-odd products of the spin-spin terms. These terms depend on the polarizations of both neutralinos, with one polarization perpendicular to the production plane. We present a detailed numerical study of the CP-sensitive observables, cross sections, and neutralino branching ratios in the Minimal Supersymmetric Standard Model with complex parameters.
\end{abstract}




\section{Introduction}

It has been pointed out that the amount of CP violation in the Standard Model (SM) is not sufficient to explain the baryon-antibaryon asymmetry of the universe [1], and that additional sources of CP violation are required [2]. Many extensions of the SM can give rise to such sources of $\mathrm{CP}$ violation. The violation of the CP symmetry is an interesting topic in its own right and deserves a diligent consideration. Supersymmetric (SUSY) extensions of the SM provide new sources of CP violation, as they include several new parameters which can be complex. For instance, in the neutralino sector of the Minimal Supersymmetric Standard Model (MSSM) two complex parameters appear, which lead to CP-violating effects in reactions involving neutralinos. These parameters are the higgsino mass parameter $\mu=|\mu| e^{i \phi_{\mu}}$, and the $\mathrm{U}(1)$ gaugino mass parameter $M_{1}=\left|M_{1}\right| e^{i \phi_{1}}$, given in the usual parametrization of modulus and phase.

These phases, on the other hand, contribute to the electric dipole moments (EDMs) of electron, neutron, and that of the atoms ${ }^{199} \mathrm{Hg}$ and ${ }^{205} \mathrm{Tl}$ [3], and it is found in general that for phases of the size $\mathcal{O}(1)$, the EDMs are beyond their experimental upper bounds. However, the extent to which the EDMs can constrain the CP phases also depends on most of the other model parameters, and thus strongly depends on the considered model, see e.g. Refs. [3,4].

In this respect the high-luminosity $e^{+} e^{-}$International Linear Collider (ILC) is considered an ideal machine to perform precision measurements, in order to determine the model parameters of the MSSM with the required accuracy [5]. In neutralino production and decay at the ILC, it has been shown which CP-even observables are well suited to access the CP-violating MSSM parameters [6,7]. However to directly prove $\mathrm{CP}$ violation in the MSSM, and to determine the CP-violating phases unambiguously, a measurement of CP-odd observables is obligatory.

In this paper, we study CP-sensitive observables in neutralino production,

$$
e^{+}+e^{-} \rightarrow \tilde{\chi}_{i}^{0}+\tilde{\chi}_{j}^{0}, \quad i, j=2,3,4, \quad i \neq j
$$

based on T-odd correlations [8] which appear in the spin-spin correlation terms of the amplitude squared. These terms involve the polarizations of both neutralinos, with one polarization perpendicular to the production plane. Such a normal polarization 
component is a genuine signal of CP violation (neglecting higher order effects due to final state interactions [8]). The polarizations of the neutralinos can be analyzed in their decays, that's why we consider the leptonic channels 1

$$
\tilde{\chi}_{i}^{0} \rightarrow \tilde{\ell}_{L, R}^{ \pm}+\ell^{\mp}, \quad \tilde{\chi}_{j}^{0} \rightarrow \tilde{\ell}_{L, R}^{ \pm}+\ell^{\prime \mp}, \quad \ell, \ell^{\prime}=e, \mu
$$

Due to angular momentum conservation, the decay distributions of the final leptons $\ell$, $\ell^{\prime}$ are correlated to each other, and thus allow us to probe the spin-spin correlations.

In a previous publication, we have analyzed in this way the CP-sensitive spin-spin correlations for chargino production and decay [10]. Other works done on CP-sensitive observables in neutralino pair production at the ILC have taken into account the decay of only one neutralino, where again the normal polarization component signals $\mathrm{CP}$ violation $[11,12]$. Even the potential of transverse beam polarizations for $\mathrm{CP}$ observables in neutralino production has been analyzed [13,14]. CP observables have also been studied in decays of neutralinos, which originate from sfermions [15].

The paper is organized as follows. In Section 2, we define the Lagrangians and complex couplings for neutralino production. In Section 3, we present the analytical formulae for the amplitude squared of neutralino production and decay. In Section 4, we identify the T-odd products in the spin-spin terms of the amplitude squared. In Section 5, we define the CP-sensitive observables which probe these terms. We present numerical results in Section 6, where we also estimate the measurability of the CPsensitive observables. We give a summary and the conclusions in Section 7.

\section{Lagrangians and complex couplings}

In the MSSM, neutralino production $e^{+} e^{-} \rightarrow \tilde{\chi}_{i}^{0} \tilde{\chi}_{j}^{0}$ proceeds via $Z$ boson exchange in the $s$-channel, and selectron $\tilde{e}_{L, R}$ exchange in the $t$ - and $u$-channels, see the Feynman diagrams in Fig. 1. The Lagrangians for production and decay are [6,16]

$$
\mathcal{L}_{Z e \bar{e}}=-\frac{g}{\cos \theta_{W}} Z_{\mu} \bar{e} \gamma^{\mu}\left[L_{e} P_{L}+R_{e} P_{R}\right] e
$$

\footnotetext{
${ }^{1}$ Note that generally parity-conserving neutralino decays, like $\tilde{\chi}_{i}^{0} \rightarrow Z \tilde{\chi}_{1}^{0}$ or $\tilde{\chi}_{i}^{0} \rightarrow h \tilde{\chi}_{1}^{0}$, would lead to vanishing CP-sensitive observables. Due to the Majorana properties of the neutralinos, the left and right neutralino couplings to the $Z$ (and Higgs) have equal absolute values, and thus all spinand spin-spin correlations would be lost, see the discussion in Ref. [9].
} 

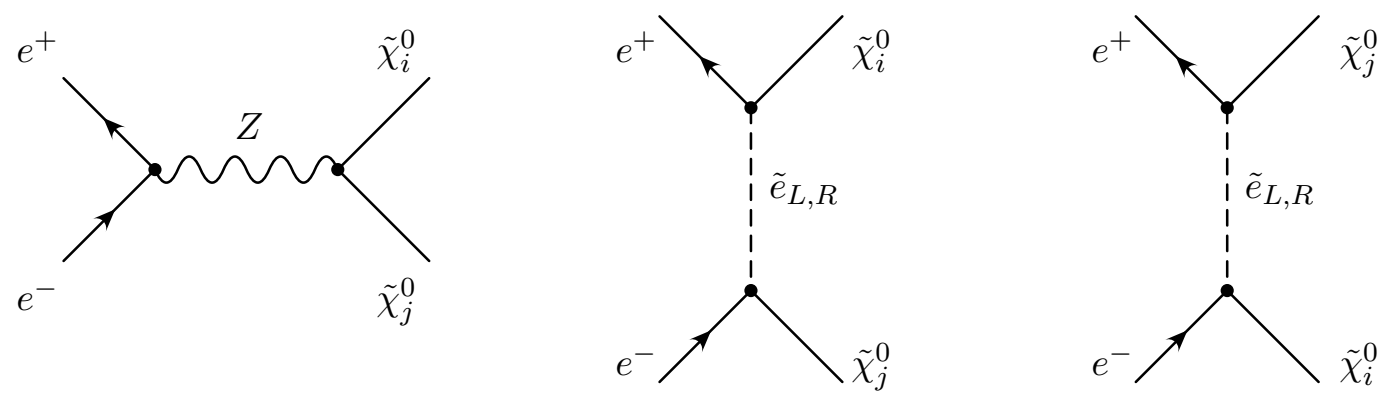

Figure 1: Feynman diagrams for neutralino production $e^{+} e^{-} \rightarrow \tilde{\chi}_{i}^{0} \tilde{\chi}_{j}^{0}[16]$.

$$
\begin{aligned}
\mathcal{L}_{Z \tilde{\chi}_{i}^{0} \tilde{\chi}_{j}^{0}} & =\frac{1}{2} \frac{g}{\cos \theta_{W}} Z_{\mu} \overline{\tilde{\chi}}_{i}^{0} \gamma^{\mu}\left[O_{i j}^{\prime \prime} P_{L}+O_{i j}^{\prime \prime} P_{R}\right] \tilde{\chi}_{j}^{0}, \quad i, j=1, \ldots, 4, \\
\mathcal{L}_{e \tilde{e} \tilde{\chi}_{i}^{0}} & =g f_{e i}^{L} \bar{e} P_{R} \tilde{\chi}_{i}^{0} \tilde{e}_{L}+g f_{e i}^{R} \bar{e} P_{L} \tilde{\chi}_{i}^{0} \tilde{e}_{R}+\text { h.c. }
\end{aligned}
$$

with $P_{L, R}=\left(1 \mp \gamma_{5}\right) / 2$. In the photino, zino, Higgsino basis the couplings are [6]

$$
\begin{aligned}
O_{i j}^{\prime \prime L} & =-\frac{1}{2}\left[\left(N_{i 3} N_{j 3}^{*}-N_{i 4} N_{j 4}^{*}\right) \cos 2 \beta+\left(N_{i 3} N_{j 4}^{*}+N_{i 4} N_{j 3}^{*}\right) \sin 2 \beta\right] \\
O_{i j}^{\prime \prime R} & =-O_{i j}^{\prime \prime} L * \\
f_{e i}^{L} & =\sqrt{2}\left[\frac{1}{\cos \theta_{W}}\left(\frac{1}{2}-\sin ^{2} \theta_{W}\right) N_{i 2}+\sin \theta_{W} N_{i 1}\right] \\
f_{e i}^{R} & =\sqrt{2} \sin \theta_{W}\left[\tan \theta_{W} N_{i 2}^{*}-N_{i 1}^{*}\right] \\
L_{e} & =\sin ^{2} \theta_{W}-\frac{1}{2}, \quad R_{e}=\sin ^{2} \theta_{W}
\end{aligned}
$$

with the weak mixing angle $\theta_{W}$, the weak coupling constant $g=e / \sin \theta_{W}, e>0$, and the ratio $\tan \beta=v_{2} / v_{1}$ of the vacuum expectation values of the two neutral Higgs fields. The neutralino couplings $O_{i j}^{\prime \prime} L, R$ and $f_{e i}^{L, R}$ contain the complex mixing elements $N_{i j}$, which diagonalize the neutralino matrix $N^{*} Y N^{\dagger}=\operatorname{diag}\left(m_{\chi_{i}^{0}}\right)$ [17], with the neutralino masses $m_{\chi_{i}^{0}}>0$. In the MSSM with CP violation, the couplings $O_{i j}^{\prime \prime} L, R$ and $f_{e i}^{L, R}$ are in general complex due to non-vanishing $\mathrm{CP}$ phases $\phi_{\mu}$ and $\phi_{1}$. Here we adopt the standard convention that a possible phase of $M_{2}$ can be absorbed by redefining the particle fields. 


\section{Cross section}

The differential cross section for neutralino production $e^{+} e^{-} \rightarrow \tilde{\chi}_{i}^{0} \tilde{\chi}_{j}^{0}$ and decay $\tilde{\chi}_{i}^{0} \rightarrow$ $\tilde{\ell}_{L, R}^{ \pm} \ell^{\mp}, \tilde{\chi}_{j}^{0} \rightarrow \tilde{\ell}_{L, R}^{\prime \pm} \ell^{\prime \mp}$, can be written

$$
\mathrm{d} \sigma=\frac{1}{2 s}|T|^{2} \text { dLips }
$$

with the center-of-mass energy $\sqrt{s}$, and the Lorentz invariant phase space element dLips, see Appendix C. The amplitude squared $|T|^{2}$ was calculated in Ref. [6] in the spin density matrix formalism 2

$$
\begin{aligned}
|T|^{2}= & 4\left|\Delta\left(\tilde{\chi}_{i}^{0}\right)\right|^{2}\left|\Delta\left(\tilde{\chi}_{j}^{0}\right)\right|^{2}\left[P D_{i} D_{j}+\sum_{a=1}^{3} \Sigma_{P}^{a} \Sigma_{D_{i}}^{a} D_{j}\right. \\
& \left.+\sum_{b=1}^{3} \Sigma_{P}^{b} \Sigma_{D_{j}}^{b} D_{i}+\sum_{a, b=1}^{3} \Sigma_{P}^{a b} \Sigma_{D_{i}}^{a} \Sigma_{D_{j}}^{b}\right],
\end{aligned}
$$

with the neutralino propagators $\Delta\left(\tilde{\chi}_{i, j}^{0}\right)=1 /\left[p_{\chi_{i, j}^{0}}^{2}-m_{\chi_{i, j}^{0}}^{2}+i m_{\chi_{i, j}^{0}} \Gamma_{\chi_{i, j}^{0}}\right]$. The amplitude squared has contributions from neutralino production $(P)$ and decay $(D)$. The terms $P$ and $D_{i}, D_{j}$ are those parts of the spin density production and decay matrices, respectively, that are independent of the polarizations of the neutralinos. The contributions $\Sigma_{P}^{a}$ and $\Sigma_{D_{i}}^{a}$ depend on the polarization basis vectors $s_{\chi_{i}}^{a}$ of neutralino $\tilde{\chi}_{i}^{0}$. Similarly, $\Sigma_{P}^{b}$ and $\Sigma_{D_{j}}^{b}$ depend on the polarization basis vectors $s_{\chi_{j}}^{b}$ of neutralino $\tilde{\chi}_{j}^{0}$. See Appendix B, Eq. (B.10) for the explicit definition of the spin vectors. We choose a coordinate frame such that $a, b=3$ denote the longitudinal polarizations, $a, b=1$ the transversal polarizations in the production plane, and $a, b=2$ the polarizations normal to the production plane. The decay terms $D_{i}, D_{j}, \Sigma_{D_{i}}^{a}$, and $\Sigma_{D_{j}}^{b}$ are given in Appendix A. The full expressions for the production terms $P, \Sigma_{P}^{a}, \Sigma_{P}^{b}$, and $\Sigma_{P}^{a b}$ can be found in Ref. [6].

The contributions to the amplitude squared which depend on the polarizations of both neutralinos are the spin-spin correlation terms $\Sigma_{P}^{a b}$. The CP-sensitive parts of the spin-spin correlation terms include one neutralino spin vector with a component perpendicular to the production plane, i.e., $a b=12,21,23,32[6]$

$$
\Sigma_{P}^{a b}(Z Z)=\frac{g^{4}}{\cos ^{4} \theta_{W}}|\Delta(Z)|^{2}\left(R_{e}^{2} c_{R}+L_{e}^{2} c_{L}\right) \operatorname{Im}\left\{O_{i j}^{\prime \prime L} O_{i j}^{\prime \prime} R *\right\} f^{a b},
$$

\footnotetext{
${ }^{2}$ For a detailed discussion of the spin density matrix formalism, we refer to Ref. [18].
} 


$$
\begin{aligned}
\Sigma_{P}^{a b}\left(Z \tilde{e}_{L}\right) & =\frac{g^{4}}{2 \cos ^{2} \theta_{W}} L_{e} c_{L} \Delta(Z)\left[\Delta_{u}^{*}\left(\tilde{e}_{L}\right)+\Delta_{t}^{*}\left(\tilde{e}_{L}\right)\right] \operatorname{Im}\left\{f_{e i}^{L} f_{e j}^{L *} O_{i j}^{\prime \prime}\right\} f^{a b}, \\
\Sigma_{P}^{a b}\left(\tilde{e}_{L} \tilde{e}_{L}\right) & =-\frac{g^{4}}{4} c_{L} \Delta_{u}\left(\tilde{e}_{L}\right) \Delta_{t}^{*}\left(\tilde{e}_{L}\right) \operatorname{Im}\left\{\left(f_{e i}^{L}\right)^{2}\left(f_{e j}^{L *}\right)^{2}\right\} f^{a b}, \\
\Sigma_{P}^{a b}\left(Z \tilde{e}_{R}\right) & =-\frac{g^{4}}{2 \cos ^{2} \theta_{W}} R_{e} c_{R} \Delta(Z)\left[\Delta_{u}^{*}\left(\tilde{e}_{R}\right)+\Delta_{t}^{*}\left(\tilde{e}_{R}\right)\right] \operatorname{Im}\left\{f_{e i}^{R} f_{e j}^{R *} O_{i j}^{\prime \prime}\right\} f^{a b}, \\
\Sigma_{P}^{a b}\left(\tilde{e}_{R} \tilde{e}_{R}\right) & =\frac{g^{4}}{4} c_{R} \Delta_{u}\left(\tilde{e}_{R}\right) \Delta_{t}^{*}\left(\tilde{e}_{R}\right) \operatorname{Im}\left\{\left(f_{e i}^{R}\right)^{2}\left(f_{e j}^{R *}\right)^{2}\right\} f^{a b} .
\end{aligned}
$$

The dependence on the longitudinal beam polarizations is given by

$$
c_{L}=\left(1-\mathcal{P}_{-}\right)\left(1+\mathcal{P}_{+}\right), \quad c_{R}=\left(1+\mathcal{P}_{-}\right)\left(1-\mathcal{P}_{+}\right)
$$

with $\mathcal{P}_{-}$and $\mathcal{P}_{+}$the degrees of longitudinal polarization of the electron and positron beam, respectively, with $-1 \leq \mathcal{P}_{ \pm} \leq 1$. Generally the contributions from the exchange of $\tilde{e}_{R}\left(\tilde{e}_{L}\right)$ are enhanced and those of $\tilde{e}_{L}\left(\tilde{e}_{R}\right)$ are suppressed for $\mathcal{P}_{-}>0, \mathcal{P}_{+}<0\left(\mathcal{P}_{-}<\right.$ $\left.0, \mathcal{P}_{+}>0\right)$. The propagators are $\Delta(Z)=i /\left(s-m_{Z}^{2}\right), \Delta_{t}\left(\tilde{e}_{L, R}\right)=i /\left(t-m_{\tilde{e}_{L, R}}^{2}\right)$, $\Delta_{u}\left(\tilde{e}_{L, R}\right)=i /\left(u-m_{\tilde{e}_{L, R}}^{2}\right)$, with $s=\left(p_{e^{-}}+p_{e^{+}}\right)^{2}, t=\left(p_{e^{-}}-p_{\chi_{j}}\right)^{2}, u=\left(p_{e^{-}}-p_{\chi_{i}}\right)^{2}[6]$.

The spin-spin correlation terms $\sum_{P}^{a b}$ in Eqs. (13)-(17) explicitly depend on the imaginary parts of the products of neutralino couplings, $\operatorname{Im}\left\{O_{i j}^{\prime \prime} O_{i j}^{\prime \prime} R *, \operatorname{Im}\left\{f_{e i}^{L} f_{e j}^{L *} O_{i j}^{\prime \prime L}\right\}\right.$, $\operatorname{Im}\left\{\left(f_{e i}^{L}\right)^{2}\left(f_{e j}^{L *}\right)^{2}\right\}, \operatorname{Im}\left\{f_{e i}^{R} f_{e j}^{R *} O_{i j}^{\prime \prime} R\right\}$, and $\operatorname{Im}\left\{\left(f_{e i}^{R}\right)^{2}\left(f_{e j}^{R *}\right)^{2}\right\}$. For $i \neq j$ they are manifestly CP-sensitive, i.e., sensitive to the phases $\phi_{\mu}$ and $\phi_{1}$ of the neutralino sector. These imaginary parts of the couplings are multiplied by T-odd factors $f^{a b}$, which we discuss in detail in the next section.

\section{T-odd products of the spin-spin correlations}

The kinematical dependence of the spin-spin correlation terms of neutralino production, Eqs. (13)-(17), is given by the T-odd function [6]

$$
\begin{aligned}
& f^{a b}=\left(p_{e^{+}} \cdot p_{\chi_{j}}\right)\left[p_{e^{-}}, p_{\chi_{i}}, s_{\chi_{i}}^{a}, s_{\chi_{j}}^{b}\right]+\left(p_{e^{-}} \cdot p_{\chi_{i}}\right)\left[p_{e^{+}}, p_{\chi_{j}}, s_{\chi_{i}}^{a}, s_{\chi_{j}}^{b}\right] \\
&+\left(p_{e^{+}} \cdot s_{\chi_{j}}^{b}\right)\left[p_{e^{-}}, p_{\chi_{i}}, p_{\chi_{j}}, s_{\chi_{i}}^{a}\right]+\left(p_{e^{-}} \cdot s_{\chi_{i}}^{a}\right)\left[p_{e^{+}}, p_{\chi_{i}}, p_{\chi_{j}}, s_{\chi_{i}}^{b}\right],
\end{aligned}
$$

with the short hand notation of the epsilon product of the four four-vectors $p_{i}$

$$
\left[p_{1}, p_{2}, p_{3}, p_{4}\right] \equiv \varepsilon_{\mu \nu \alpha \beta} p_{1}^{\mu} p_{2}^{\nu} p_{3}^{\alpha} p_{4}^{\beta}, \quad \text { with } \quad \varepsilon_{0123}=-1 .
$$


Since each of the spacial components of the four-momenta or spins changes sign under a naive time transformation, $t \rightarrow-t$, the epsilon product, and thus the function $f^{a b}$, is T-odd. In Appendix $\mathrm{B}$, we give $f^{a b}$ also in the laboratory system.

In order to identify the T-odd products which appear in the spin-spin correlations of production and decay, we analyze the corresponding terms of the amplitude squared, Eq. (12), in more detail

$$
|T|^{2} \propto \sum_{a, b=1}^{3} \Sigma_{P}^{a b} \Sigma_{D_{i}}^{a} \Sigma_{D_{j}}^{b} \propto \sum_{a, b=1}^{3} f^{a b} \cdot\left(p_{\ell} \cdot s_{\chi_{i}}^{a}\right) \cdot\left(p_{\ell^{\prime}} \cdot s_{\chi_{j}}^{b}\right)
$$

where the scalar products $\left(p_{\ell} \cdot s_{\chi_{i}}^{a}\right)$ and $\left(p_{\ell^{\prime}} \cdot s_{\chi_{j}}^{b}\right)$ stem from $\Sigma_{D_{i}}^{a}$ and $\Sigma_{D_{j}}^{b}$, respectively, see Eqs. (A.2) or (A.4) in Appendix A. Using the explicit expression for $f^{a b}$, Eq. (19), and the completeness relation for the neutralino spin vectors, Eq. (B.11), the righthand side of of the second equation in Eq. (21) can be written as

$$
\begin{aligned}
\mathcal{O}_{T} & =\left(p_{e^{+}} \cdot p_{\chi_{j}}\right)\left[p_{e^{-}}, p_{\chi_{i}}, p_{\ell}, p_{\ell^{\prime}}\right]+\left(p_{e^{-}} \cdot p_{\chi_{i}}\right)\left[p_{e^{+}}, p_{\chi_{j}}, p_{\ell}, p_{\ell^{\prime}}\right] \\
& +\left(p_{e^{+}} \cdot p_{\ell^{\prime}}\right)\left[p_{e^{-}}, p_{\chi_{i}}, p_{\chi_{j}}, p_{\ell}\right]+\left(p_{e^{-}} \cdot p_{\ell}\right)\left[p_{e^{+}}, p_{\chi_{i}}, p_{\chi_{j}}, p_{\ell^{\prime}}\right] .
\end{aligned}
$$

We have now identified the CP-sensitive terms of the neutralino spin-spin correlations. They are proportional to the T-odd product $\mathcal{O}_{T}$, Eq. (22), which can now be used to define various $\mathrm{CP}$ asymmetries and $\mathrm{CP}$ observables in neutralino production and decay. Due to their similar kinematical dependence, the definition of CP observables is analogous to those in chargino production and decay, see Ref. [10].

\section{CP-sensitive observables}

In this Section, we define various CP-sensitive observables, which depend on the Todd parts of the spin-spin correlations for neutralino production and decay. For an operator $\mathcal{O}$, we define its expectation value by [10]

$$
\langle\mathcal{O}\rangle=\frac{\int \mathcal{O}|T|^{2} \mathrm{dLips}}{\int|T|^{2} \mathrm{dLips}}=\frac{1}{\sigma} \int \mathcal{O} \frac{\mathrm{d} \sigma}{\mathrm{dLips}} \mathrm{dLips}
$$

If the operator $\mathcal{O}$ is chosen of the form like the T-odd terms $\mathcal{O}_{T}$, Eq. (22), the CPsensitive parts of the spin-spin correlations in neutralino production can be projected 
out

$$
\langle\mathcal{O}\rangle=\frac{\int \mathcal{O} \Sigma_{P}^{a b} \Sigma_{D_{i}}^{a} \Sigma_{D_{j}}^{b} \mathrm{dLips}}{\int P D_{i} D_{j} \mathrm{dLips}}
$$

with an implicit sum over $(a, b)=(1,2),(2,1),(2,3),(3,2)$. In the numerator remain only the CP-sensitive parts of the spin-spin terms of the amplitude squared. Only they contain the T-odd product $\mathcal{O}$. In the denominator, all spin- and spin-spin correlation terms vanish, and only the spin-independent part $P D_{i} D_{j}$ contributes. Note that for the phase space element dLips in Eq. (24), we have already used the narrow width approximation for the propagators, see Eq. (C.22).

In general the largest observables are obtained by using an operator $\mathcal{O}$, which exactly matches the kinematical dependence of the CP-sensitive terms in the amplitude squared, that is $\mathcal{O}=\mathcal{O}_{T}$, Eq. (22). In the literature, this technique is sometimes referred to optimal observables [19]. Thus for the operator $\mathcal{O}_{T}$ we define the two CP-sensitive observables

$$
\left\langle\mathcal{O}_{T}\right\rangle \quad \text { and } \quad \mathcal{A}_{T}=\left\langle\operatorname{Sgn}\left(\mathcal{O}_{T}\right)\right\rangle
$$

Neglecting higher order effects due to final state interactions [8], the observable $\mathcal{A}_{T}$ is a CP asymmetry. It is the expectation value for the sign of the T-odd product $\mathcal{O}_{T}$, and can be written as

$$
\mathcal{A}_{T}=\frac{N_{+}-N_{-}}{N_{+}+N_{-}}
$$

the difference of the number of events with positive $\left(N_{+}\right)$and negative $\left(N_{-}\right)$sign of $\mathcal{O}_{T}$, normalized by the total number of events $N=N_{+}+N_{-}$. On the other hand, $\left\langle\mathcal{O}_{T}\right\rangle$ is the expectation value of the momentum configuration $\mathcal{O}_{T}$ itself for the event sample.

Two further T-odd products were considered in Ref. [10]. One product is obtained from $\mathcal{O}_{T}$, Eq. (22) , in replacing the four-momenta by the (normalized) threemomentum vectors in the center-of-mass system, see Appendix B,

$$
\widehat{\mathcal{O}}_{T}=\left(\hat{p}_{e^{-}} \cdot \hat{p}_{\ell^{\prime}}\right) \hat{p}_{e^{-}} \cdot\left(\hat{p}_{\chi_{j}} \times \hat{p}_{\ell}\right)+\left(\hat{p}_{e^{-}} \cdot \hat{p}_{\ell}\right) \hat{p}_{e^{-}} \cdot\left(\hat{p}_{\chi_{j}} \times \hat{p}_{\ell^{\prime}}\right)
$$


with $\hat{p}=\vec{p} /|\vec{p}|$. In contrast to $\mathcal{O}_{T}$, Eq. (22), this product does not involve the energies of the neutralinos and leptons. For the operator $\widehat{\mathcal{O}}_{T}$, we again define two $\mathrm{CP}$-sensitive observables

$$
\left\langle\widehat{\mathcal{O}}_{T}\right\rangle \quad \text { and } \quad \hat{\mathcal{A}}_{T}=\left\langle\operatorname{Sgn}\left(\widehat{\mathcal{O}}_{T}\right)\right\rangle
$$

Since both T-odd products $\mathcal{O}_{T}$ and $\widehat{\mathcal{O}}_{T}$ include the neutralino momentum $p_{\chi_{i}}$ and/or $p_{\chi_{j}}$, their experimental reconstruction is required. For the subsequent two-body decays of the neutralinos which we consider here, the neutralino momentum three-vectors can be reconstructed up to a sign ambiguity in their second component, if the masses of the involved particles are known, see for example Ref. [13].

A T-odd product which does not depend on the neutralino momenta is obtained by substituting on the right hand side of Eq. (27) the neutralino three-momenta by the corresponding decay lepton three-momenta $\vec{p}_{\chi_{i}} \rightarrow \vec{p}_{\ell}$ and $\vec{p}_{\chi_{j}} \rightarrow \vec{p}_{\ell^{\prime}}[10]$,

$$
\widehat{\mathcal{O}}_{T}^{\prime}=\hat{p}_{e^{-}} \cdot\left(\hat{p}_{\ell}+\hat{p}_{\ell^{\prime}}\right) \hat{p}_{e^{-}} \cdot\left(\hat{p}_{\ell} \times \hat{p}_{\ell^{\prime}}\right) .
$$

Also for $\widehat{\mathcal{O}}_{T}^{\prime}$ we define a CP-sensitive observable and its corresponding asymmetry,

$$
\left\langle\widehat{\mathcal{O}}_{T}^{\prime}\right\rangle \quad \text { and } \quad \hat{\mathcal{A}}_{T}^{\prime}=\left\langle\operatorname{Sgn}\left(\widehat{\mathcal{O}}_{T}^{\prime}\right)\right\rangle
$$

Thus, depending on the type of correlation used, two classes of CP observables can be defined; one class requires the reconstruction of the neutralino momenta, the other class not. However, as we will show in the numerical section, the largest observables are obtained if indeed the neutralino momenta can be reconstructed.

\subsection{Relative signs of the CP observables}

Each of the above defined $\mathrm{CP}$ observables depends in principle on the various decay channels of the two neutralinos. For each neutralino, these are

$$
\begin{aligned}
\tilde{\chi}_{k}^{0} & \rightarrow \tilde{\ell}_{R}^{+}+\ell^{-}, \\
& \rightarrow \tilde{\ell}_{R}^{-}+\ell^{+},
\end{aligned}
$$


Table 1: Relative signs of the CP-sensitive observables for different decay combinations of neutralino $\tilde{\chi}_{i}^{0} \rightarrow \tilde{\ell}_{L(R)}^{ \pm} \ell^{\mp}$ (top row), and neutralino $\tilde{\chi}_{j}^{0} \rightarrow \tilde{\ell}_{L(R)}^{ \pm} \ell^{\prime}$ (left column).

\begin{tabular}{c|c|c|c|c} 
& $\tilde{\ell}_{R}^{+}$ & $\tilde{\ell}_{R}^{-}$ & $\tilde{\ell}_{L}^{+}$ & $\tilde{\ell}_{L}^{-}$ \\
\hline$\tilde{\ell}_{R}^{+}$ & + & - & - & + \\
\hline$\tilde{\ell}_{R}^{-}$ & - & + & + & - \\
\hline$\tilde{\ell}_{L}^{+}$ & - & + & + & - \\
\hline$\tilde{\ell}_{L}^{+}$ & + & - & - & +
\end{tabular}

for $\ell=e, \mu$, and also

$$
\begin{aligned}
\tilde{\chi}_{k}^{0} & \rightarrow \tilde{\ell}_{L}^{+}+\ell^{-}, \\
& \rightarrow \tilde{\ell}_{L}^{-}+\ell^{+},
\end{aligned}
$$

if the decay into the (usually) heavier left slepton is kinematically allowed. However, only the sign of the CP observable changes, depending on the charge and the type (L or R) of the two decay sleptons, for an overview see Table 1. The reason is that the signs of the corresponding two neutralino decay terms, $\Sigma_{D_{i}}^{a}$ and $\Sigma_{D_{j}}^{b}$, only depend on the charge and the type of the two decay sleptons, see Eqs. (A.2) and (A.4), respectively. The absolute value of an observable is independent of the particular decay channels, since the absolute values of the couplings $\left|f_{\ell i}^{L, R}\right|$ or $\left|f_{\ell j}^{L, R}\right|$ of the decay sleptons, as well as their masses, cancel in the numerator and denominator of Eq. (24). This means in turn that we have to distinguish from which neutralino $\tilde{\chi}_{i}^{0}$ or $\tilde{\chi}_{j}^{0}$ the final state leptons $\ell$ and $\ell^{\prime}$ originate. Without that information, the contributions to the CP observables from the final leptons with charge combinations $\ell^{-} \ell^{\prime-}$ and $\ell^{+} \ell^{\prime+}$ would identically cancel those contribution from $\ell^{+} \ell^{\prime-}$ and $\ell^{-} \ell^{\prime+}$. Furthermore if also the decay into $\tilde{\ell}_{L}$ is kinematically possible, the type of the sleptons, $\tilde{\ell}_{L}$ or $\tilde{\ell}_{R}$, into which the neutralinos decay, has to be determined. Such a discrimination can in principle be accomplished by using the different energy distributions of the decay leptons, since their kinematical limits depend on the mass difference of the decaying neutralino and slepton. Certainly the feasibility of such an event reconstruction can only be answered by a detailed experimental analysis, which is however beyond the scope of the present work. 


\subsection{Theoretical statistical significances}

We have defined various kinds of CP-sensitive observables, which are based on the different T-odd products $\mathcal{O}=\mathcal{O}_{T}, \widehat{\mathcal{O}}_{T}, \widehat{\mathcal{O}}_{T}^{\prime}$. They either include $\left(\mathcal{O}_{T}, \widehat{\mathcal{O}}_{T}\right)$ or not include $\left(\widehat{\mathcal{O}}_{T}^{\prime}\right)$ the neutralino momentum. In order to be able to compare these observables quantitatively, we define their theoretical statistical significances. A comparison of the numerical values of $\langle\mathcal{O}\rangle$ and $\mathcal{A}=\langle\operatorname{Sgn}(\mathcal{O})\rangle$ alone cannot be used to decide which observable is more sensitive to the $\mathrm{CP}$ phases. In addition, we are sometimes facing situations where large CP observables and asymmetries correspond to processes with small neutralino production cross sections or branching ratios, and vice versa. Such effects can be considered by combining both the CP observable and the cross section into one statistical quantity.

We define the theoretical statistical significance of the CP observable $\langle\overline{\mathcal{O}}\rangle$, where $\overline{\mathcal{O}}=\mathcal{O}$, or $\overline{\mathcal{O}}=\operatorname{Sgn}(\mathcal{O})$, by $[13,20]$

$$
S[\overline{\mathcal{O}}]=\sqrt{N} \frac{|\langle\overline{\mathcal{O}}\rangle|}{\sqrt{\left\langle\overline{\mathcal{O}}^{2}\right\rangle}}
$$

For neutralino production and decay the number of events is

$$
\begin{aligned}
N=F_{N} \times \mathcal{L} \times \sigma\left(e^{+} e^{-} \rightarrow \tilde{\chi}_{i}^{0} \tilde{\chi}_{j}^{0}\right) \times & {\left[\mathrm{BR}\left(\tilde{\chi}_{i}^{0} \rightarrow \tilde{e}_{R}^{+} e^{-}\right) \times \operatorname{BR}\left(\tilde{\chi}_{j}^{0} \rightarrow \tilde{e}_{R}^{+} e^{-}\right)\right.} \\
& +\mathrm{BR}\left(\tilde{\chi}_{i}^{0} \rightarrow \tilde{e}_{L}^{+} e^{-}\right) \times \operatorname{BR}\left(\tilde{\chi}_{j}^{0} \rightarrow \tilde{e}_{L}^{+} e^{-}\right) \\
& +\mathrm{BR}\left(\tilde{\chi}_{i}^{0} \rightarrow \tilde{e}_{R}^{+} e^{-}\right) \times \operatorname{BR}\left(\tilde{\chi}_{j}^{0} \rightarrow \tilde{e}_{L}^{+} e^{-}\right) \\
& \left.+\mathrm{BR}\left(\tilde{\chi}_{i}^{0} \rightarrow \tilde{e}_{L}^{+} e^{-}\right) \times \operatorname{BR}\left(\tilde{\chi}_{j}^{0} \rightarrow \tilde{e}_{R}^{+} e^{-}\right)\right],
\end{aligned}
$$

with the integrated luminosity $\mathcal{L}$. The combinatorial factor $F_{N}$ takes into account all possible neutralino decays into sleptons with different flavors and charges. We assume that the branching ratios of the neutralinos do not depend on them, i.e., $\operatorname{BR}\left(\tilde{\chi}_{k}^{0} \rightarrow \tilde{e}_{n}^{+} e^{-}\right)=\operatorname{BR}\left(\tilde{\chi}_{k}^{0} \rightarrow \tilde{e}_{n}^{-} e^{+}\right)=\operatorname{BR}\left(\tilde{\chi}_{k}^{0} \rightarrow \tilde{\mu}_{n}^{+} \mu^{-}\right)=\operatorname{BR}\left(\tilde{\chi}_{k}^{0} \rightarrow \tilde{\mu}_{n}^{-} \mu^{+}\right)$, for $n=L$ and $R$. The combinatorial factor is thus $F_{N}=4 \times 4=16$, if we sum the two lepton flavors $e, \mu$ and the two charges, $\tilde{\ell}_{n}^{+}$and $\tilde{\ell}_{n}^{-}$.

The statistical significance $S$ is equal to the number of standard deviations to which the corresponding CP observable can be determined to be non-zero over statistical fluctuations. For example, $S=1$ implies a measurement at the statistical $68 \%$ confidence 
level, assuming an ideal detector, and full reconstruction of signal and background. Thus our definition of $S$ is theoretically motivated, and can only be regarded as as an upper bound on the confidence level which at best can be obtained. In order to give realistic values of the statistical significances, a detailed experimental study would be required, which is however beyond the scope of the present work.

\section{$6 \quad$ Numerical results}

We present numerical results for the CP-sensitive observables and asymmetries for neutralino production $e^{+} e^{-} \rightarrow \tilde{\chi}_{2}^{0} \tilde{\chi}_{3}^{0}$, and decay $\tilde{\chi}_{2}^{0} \rightarrow \tilde{\ell}_{R}^{ \pm} \ell^{\mp}, \tilde{\chi}_{3}^{0} \rightarrow \tilde{\ell}_{R}^{\prime \pm} \ell^{\prime \mp}$, for $\ell, \ell^{\prime}=$ $e, \mu$. We will study the dependence of the CP observables on the phases and moduli of the higgsino and $\mathrm{U}(1)$ gaugino mass parameters $\mu=|\mu| e^{i \phi_{\mu}}$ and $M_{1}=\left|M_{1}\right| e^{i \phi_{1}}$, respectively, in the framework of the general MSSM. In this model the restrictions on the phases from the electron and neutron EDMs are less severe compared to the constrained MSSM [4]. Thus we will not take the EDMs into account, and show the full phase dependence of the observables.

The results are calculated with a center-of-mass energy of $\sqrt{s}=500 \mathrm{GeV}$. We choose longitudinal beam polarizations $\left(\mathcal{P}_{-}, \mathcal{P}_{+}\right)=(0.9,-0.6)$, which enhance the $\tilde{e}_{R}$ exchange contribution. The feasibility of measuring the observables also depends on the neutralino production cross section and decay branching ratios, which we discuss in detail. For a comparison of the CP observables, and for giving an upper bound on the confidence levels, we also present a theoretical estimate of their statistical significances.

For the calculation of the neutralino decay widths and branching ratios, we include the two-body decays [12]

$$
\tilde{\chi}_{i}^{0} \rightarrow \tilde{\ell}_{n}+\ell, \quad \tilde{\nu}_{\ell}+\nu_{\ell}, \quad Z+\tilde{\chi}_{m}^{0}, \quad h+\tilde{\chi}_{m}^{0}, \quad W^{ \pm}+\tilde{\chi}_{k}^{\mp},
$$

with $m<i ; k=1,2 ; n=R, L$ for $\ell=e, \mu$, and $n=1,2$ for $\ell=\tau$. We neglect neutralino three-body decays. We use the GUT inspired relation $\left|M_{1}\right|=5 / 3 M_{2} \tan ^{2} \theta_{W}$, such that the dependence of the CP observables on the modulus of $M_{1}$ is investigated by using $M_{2}$. In order to reduce the number of free parameters further, we parametrize the slepton masses by $M_{2}$, and $m_{0}=80 \mathrm{GeV}$ fixed, which enter in the approximate solutions to the renormalization group equations, see Appendix A. We take stau mix- 
Table 2: Benchmark scenario for $e^{+} e^{-} \rightarrow \tilde{\chi}_{2}^{0} \tilde{\chi}_{3}^{0}$, and decay $\tilde{\chi}_{2}^{0} \rightarrow \tilde{\ell}_{R}^{ \pm} \ell^{\mp}, \tilde{\chi}_{3}^{0} \rightarrow \tilde{\ell}_{R}^{ \pm} \ell^{\prime \mp}$, for $\ell, \ell^{\prime}=e, \mu$, at $\sqrt{s}=500 \mathrm{GeV}$ with beam polarizations $\left(\mathcal{P}_{-}, \mathcal{P}_{+}\right)=(0.9,-0.6)$.

\begin{tabular}{cccccc}
\hline$M_{2}$ & $|\mu|$ & $\phi_{\mu}$ & $\phi_{1}$ & $\tan \beta$ & $m_{0}$ \\
\hline $270 \mathrm{GeV}$ & $200 \mathrm{GeV}$ & 0 & $0.5 \pi$ & 3 & $80 \mathrm{GeV}$ \\
\hline
\end{tabular}

Table 3: SUSY masses, neutralino branching ratios and production cross section, for the benchmark scenario. The branching ratios are summed over $\ell=e, \mu$ and both slepton charges.

\begin{tabular}{|l|l|l|}
\hline$m_{\chi_{1}^{0}}=121 \mathrm{GeV}$ & $m_{\tilde{e}_{R}}=157 \mathrm{GeV}$ & $\operatorname{BR}\left(\tilde{\chi}_{2}^{0} \rightarrow \tilde{\ell}_{R} \ell\right)=66 \%$ \\
\hline$m_{\chi_{2}^{0}}=171 \mathrm{GeV}$ & $m_{\tilde{e}_{L}}=256 \mathrm{GeV}$ & $\operatorname{BR}\left(\tilde{\chi}_{2}^{0} \rightarrow \tilde{\tau}_{1} \tau\right)=34 \%$ \\
\hline$m_{\chi_{3}^{0}}=207 \mathrm{GeV}$ & $m_{\tilde{\tau}_{1}}=157 \mathrm{GeV}$ & $\operatorname{BR}\left(\tilde{\chi}_{3}^{0} \rightarrow \tilde{\ell}_{R} \ell\right)=66 \%$ \\
\hline$m_{\chi_{1}^{ \pm}}=158 \mathrm{GeV}$ & $m_{\tilde{\tau}_{2}}=256 \mathrm{GeV}$ & $\operatorname{BR}\left(\tilde{\chi}_{3}^{0} \rightarrow \tilde{\tau}_{1} \tau\right)=34 \%$ \\
\hline$m_{\chi_{2}^{ \pm}}=318 \mathrm{GeV}$ & $m_{\tilde{\nu}}=246 \mathrm{GeV}$ & $\sigma\left(e^{+} e^{-} \rightarrow \tilde{\chi}_{2}^{0} \tilde{\chi}_{3}^{0}\right)=79 \mathrm{fb}$ \\
\hline
\end{tabular}

ing into account, and fix the trilinear scalar coupling parameter $A_{\tau}=250 \mathrm{GeV}$. For the SM parameters, we use $\sin ^{2} \theta_{W}=0.2315, m_{W}=80.41 \mathrm{GeV}, m_{Z}=91.187 \mathrm{GeV}$, $\alpha=7.8125 \times 10^{-3}$.

The CP-sensitive neutralino coupling factors in Eqs. (13)-(17) are zero for $i=j$, or vanishing phases $\phi_{\mu}$ and $\phi_{1}$. They are largest for $\phi_{1}=0.5 \pi$ (or $1.5 \pi$ ), and for a strong gaugino-higgsino mixing $M_{2} \approx|\mu|$. Furthermore we find that a small value of $\tan \beta$ is preferred to have large CP observables and large significances. Therefore we center our numerical discussion around a scenario with $\tan \beta=3$ and a strong gaugino-higgsino mixing. The parameters are summarized in Tab. 2. The corresponding particle masses, branching ratios, and the cross section are listed in Tab. 3. For this scenario, we first analyze the phase dependence of the CP observables, and then study their dependence on $|\mu|$ and $M_{2}$. 


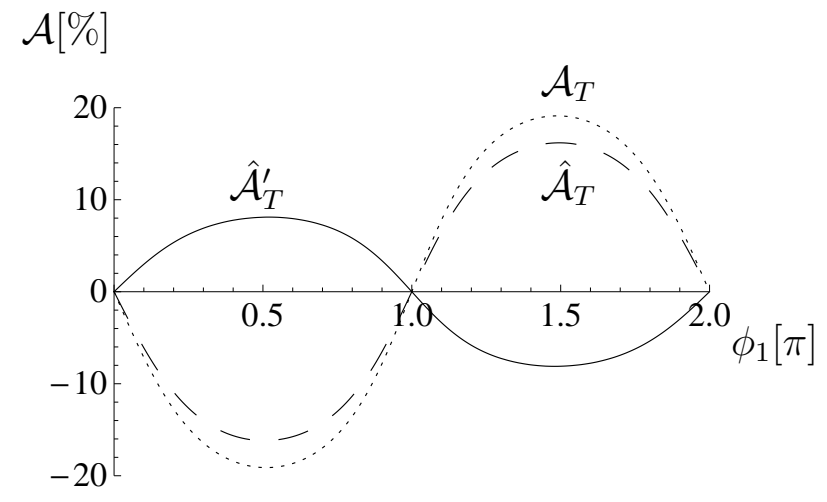

(a)
$\mathcal{A}[\%]$

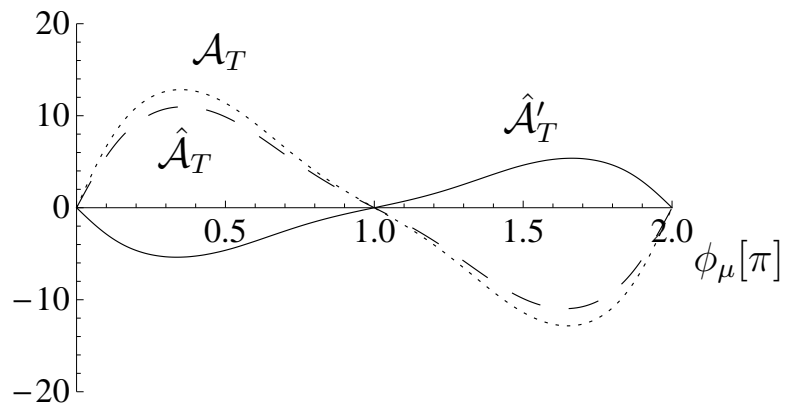

(b)

Figure 2: Dependence of the CP asymmetries $\mathcal{A}_{T}$ (dotted), $\hat{\mathcal{A}}_{T}$ (dashed), and $\hat{\mathcal{A}}_{T}^{\prime}$ (solid), (a) on the phase $\phi_{1}$ with $\phi_{\mu}=0$, and (b) on the phase $\phi_{\mu}$ with $\phi_{1}=\pi$, and the other parameters as defined in Tab. 2 .

\subsection{Phase dependence}

In Fig. 2(a), we show the $\phi_{1}$ dependence of the CP asymmetries $\mathcal{A}_{T}(25), \hat{\mathcal{A}}_{T}(28)$, and $\hat{\mathcal{A}}_{T}^{\prime}(\underline{30})$. The asymmetries vanish at $\phi_{1}=0, \pi, 2 \pi$, where the neutralino couplings are real. They obtain largest values at $\phi_{1} \approx 0.5 \pi$ and $\phi_{1} \approx 1.5 \pi$ of about $\mathcal{A}_{T}= \pm 19 \%$, $\hat{\mathcal{A}}_{T}= \pm 16 \%$, and $\hat{\mathcal{A}}_{T}^{\prime}= \pm 8 \%$. In Fig. 2(b), we show the same asymmetries as a function of $\phi_{\mu}$, setting $\phi_{1}=\pi$. They show a similar behavior, and again $\mathcal{A}_{T}$ attains the largest values of all three asymmetries. We do not present plots of the corresponding observables, since they show similar phase dependences as their corresponding asymmetries. They obtain values of $\left\langle\mathcal{O}_{T}\right\rangle=-2.56 \times 10^{11} \mathrm{GeV}^{6},\left\langle\widehat{\mathcal{O}}_{T}\right\rangle=-0.062$, and $\left\langle\widehat{\mathcal{O}}_{T}^{\prime}\right\rangle=0.027$, for the scenario defined in Tab. 2.

In order to compare now the $\mathrm{CP}$ asymmetries $\mathcal{A}$ with their corresponding $\mathrm{CP}$ observables $\langle\mathcal{O}\rangle$, we show their theoretical significances $S$ as a function of $\phi_{1}$ in Fig. 3 . First we observe that the observables, Fig. 3(b), have generally larger significances than their counterpart asymmetries, Fig. 3(a). Secondly, the observables and asymmetries which are based on the T-odd products $\mathcal{O}_{T}$ and $\widehat{\mathcal{O}}_{T}$, which include the neutralino momentum, have the largest significances. They would be best suited for measuring $\mathrm{CP}$ phases in the neutralino spin-spin correlations. The significance of $\left\langle\mathcal{O}_{T}\right\rangle$ is twice as large as that of $\left\langle\widehat{\mathcal{O}}_{T}^{\prime}\right\rangle$. However, for their measurement a reconstruction of the neutralino momenta, i.e., the production plane is necessary, which will be experimentally more involved. The need to only reconstruct the final state leptons might be an 


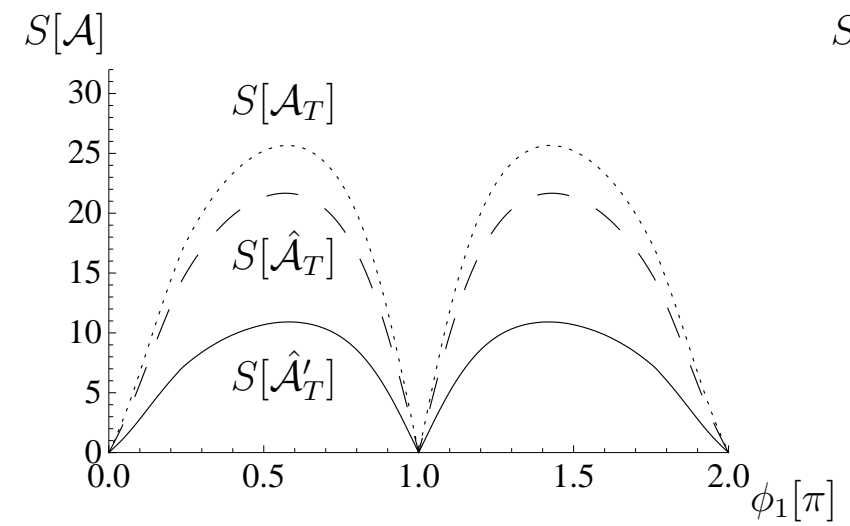

(a)

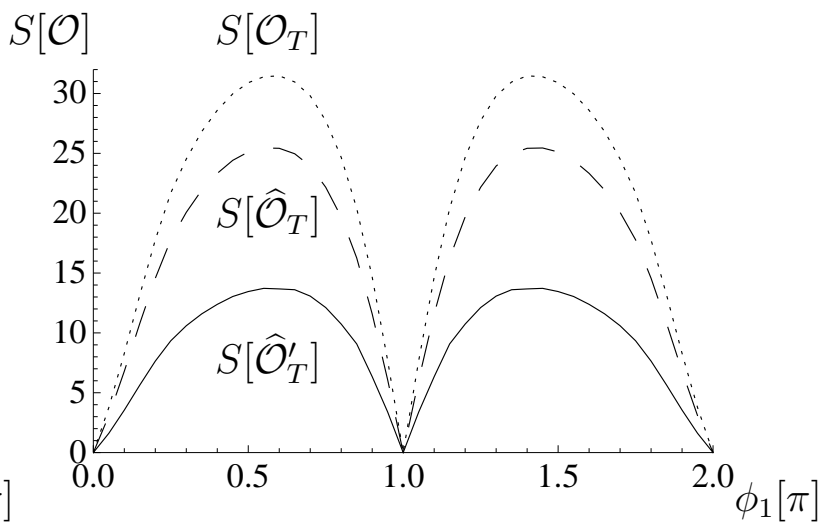

(b)

Figure 3: Phase-dependence of the significances of (a) the asymmetries $\mathcal{A}_{T}$ (dotted), $\hat{\mathcal{A}}_{T}$ (dashed), $\hat{\mathcal{A}}_{T}^{\prime}$ (solid), and (b) of the observables $\left\langle\mathcal{O}_{T}\right\rangle$ (dotted), $\left\langle\widehat{\mathcal{O}}_{T}\right\rangle$ (dashed), $\left\langle\widehat{\mathcal{O}}_{T}^{\prime}\right\rangle$ (solid), with an integrated luminosity of $\mathcal{L}=500 \mathrm{fb}^{-1}$, for the scenario as defined in Tab. 2.

advantage in a realistic experimental environment. However, a detailed investigation which observable will be best suited can only be answered by a thorough experimental analysis, which is beyond the scope of the present work. In order to further illustrate the different magnitudes of the asymmetries $\mathcal{A}_{T}$ and $\hat{\mathcal{A}}_{T}^{\prime}$, we show them and the corresponding significances as a function of the phases $\phi_{\mu}$ and $\phi_{1}$ in Fig. 4 .

Finally, it should be noted that a measurement of observables which depend only on $\phi_{\mu}$ will be helpful to disentangle CP-violating effects in the neutralino system, which could originate both from $\phi_{1}$ and $\phi_{\mu}$. This could be possible by investigating $\mathrm{CP}$ observables in the chargino system $[10,21]$ which solely depend on $\phi_{\mu}$. Finally a global fit of CP-even [6,7] and CP-odd [11-14] observables in the neutralino system could allow for a complete determination of the phases. 
$\mathcal{A}_{T}$ in $\%$

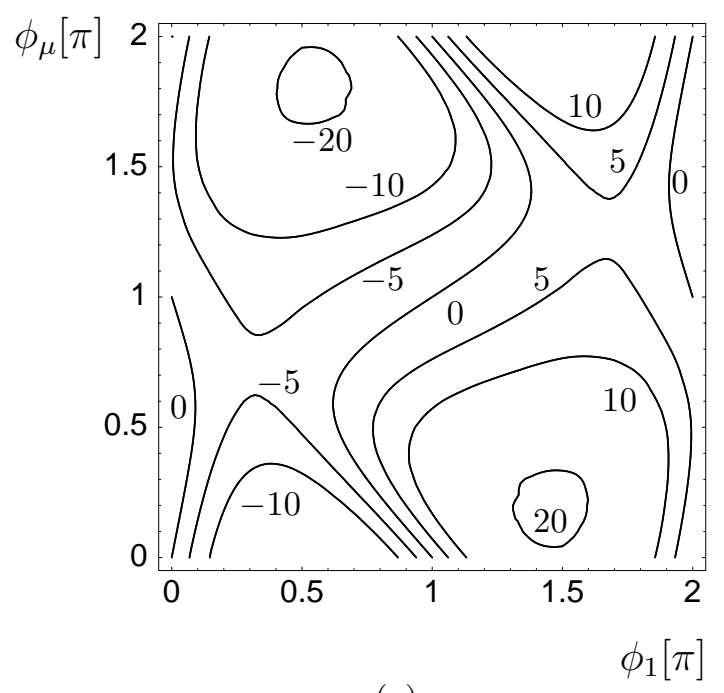

(a)

\section{$\hat{\mathcal{A}}_{T}^{\prime}$ in $\%$}

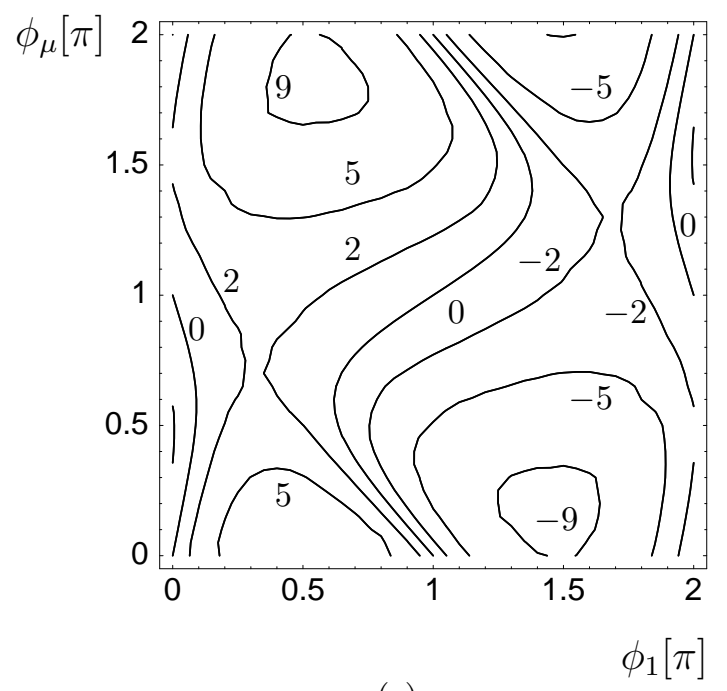

(c)

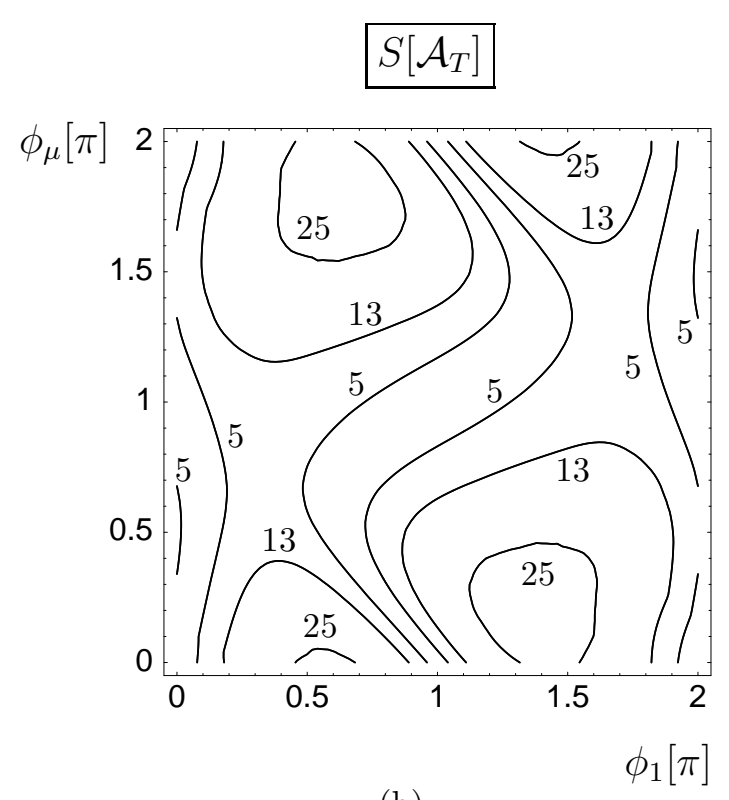

(b)

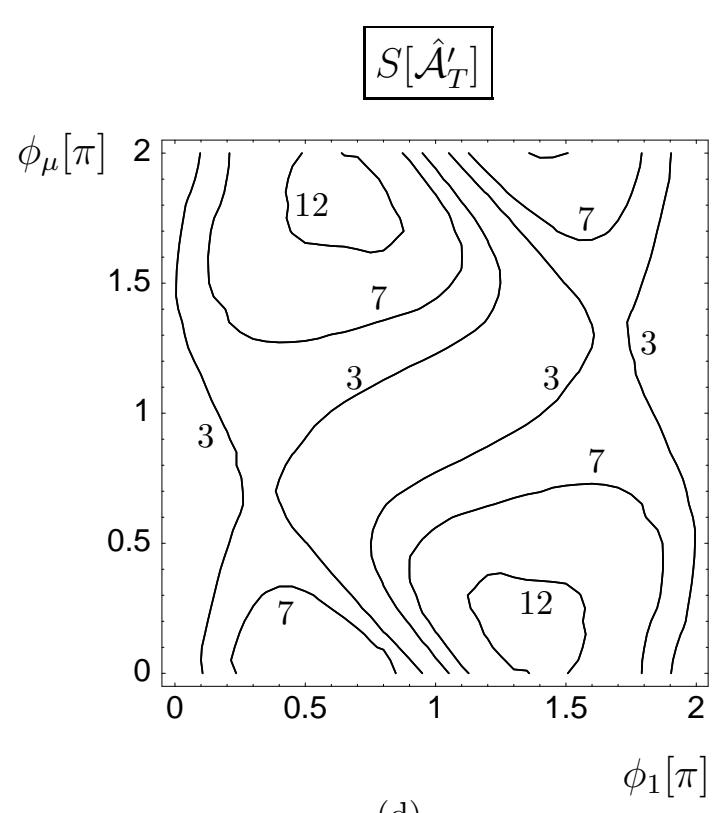

(d)

Figure 4: Contour lines of the asymmetries $\mathcal{A}_{T}$ and $\hat{\mathcal{A}}_{T}^{\prime}$, and their statistical significances in the $\phi_{1}-\phi_{\mu}$ plane, with an integrated luminosity of $\mathcal{L}=500 \mathrm{fb}^{-1}$, for the scenario as defined in Tab. 2. 


\section{2 $\mu$ and $M_{2}$ dependence}

In order to estimate the significances of the CP-sensitive observables in a larger region of the parameter space, we now analyze the neutralino cross sections, branching ratios and, as an example, the asymmetry $\mathcal{A}_{T}(25)$ in the $|\mu|-M_{2}$ plane. The other parameters are as given in Tab. 2 .

In Figs. 5(a) and 5(b), we show the neutralino branching ratios which are summed over both lepton flavors $\ell=e, \mu$ and charges, i.e., $\operatorname{BR}\left(\tilde{\chi}_{k}^{0} \rightarrow \tilde{\ell}_{R} \ell\right)=4 \times \operatorname{BR}\left(\tilde{\chi}_{k}^{0} \rightarrow\right.$ $\tilde{e}_{R}^{+} e^{-}$), for $k=2,3$. In the gray shaded area, the chargino mass is $m_{\chi_{1}^{ \pm}}<100 \mathrm{GeV}$, and thus near or below the exclusion limit of LEP2 [22]. In region $A$, the neutralinos are below the decay threshold, $m_{\chi_{2,3}^{0}}<m_{\tilde{\ell}_{R}}$, and thus the corresponding two-body decays are closed. The neutralino $\tilde{\chi}_{2}^{0}$ is always lighter than $\tilde{\ell}_{L}$ in the shown region of the $|\mu|-M_{2}$ plane. We find that the $\tilde{\chi}_{3}^{0}$ branching ratio into left sleptons is smaller than $\operatorname{BR}\left(\tilde{\chi}_{3}^{0} \rightarrow \tilde{\ell}_{L} \ell\right)<1 \%$. In Fig. $5(\mathrm{a})$ and $5(\mathrm{~b})$, the decay channels into the lightest Higgs and $Z$ bosons open to the right of the dashed lines, which indicate the kinematical limit $m_{\chi_{2,3}^{0}}=m_{\chi_{1}^{0}}+m_{Z}$, respectively. However, these channels would lead to vanishing CP observables, due to the Majorana properties of the Higgs and $Z$ boson couplings to the neutralinos, as discussed in the introduction. Along the dotted contour in Fig. $5(\mathrm{~b})$, the decay channel $\tilde{\chi}_{3}^{0} \rightarrow W^{ \pm} \tilde{\chi}_{1}^{\mp}$ opens, which also considerably reduces $\operatorname{BR}\left(\tilde{\chi}_{3}^{0} \rightarrow \tilde{\ell}_{R} \ell\right)$ to the right of that contour, for $|\mu| \gtrsim M_{2}$. The neutralino $\tilde{\chi}_{2}^{0}$ and $\tilde{\chi}_{3}^{0}$ branching ratios into staus become larger than those into selectrons for $|\mu| \gtrsim M_{2}$. If the tau momenta can be reconstructed, these decay channels can also be used to measure the CP observables. However due to stau mixing, the observables will be reduced compared to the decays into selectrons or smuons, see the discussion in Ref. [9].

The neutralino production cross section $\sigma_{23}=\sigma\left(e^{+} e^{-} \rightarrow \tilde{\chi}_{2}^{0} \tilde{\chi}_{3}^{0}\right)$ is shown in Fig. 5(c). It reaches values up to $130 \mathrm{fb}$ for $M_{2} \approx 250 \mathrm{GeV}$ and $|\mu| \approx 150 \mathrm{GeV}$. In the region $B$, the neutralinos are too heavy and above the production threshold, $m_{\chi_{2}^{0}}+m_{\chi_{3}^{0}}>\sqrt{s}=500 \mathrm{GeV}$. The combined cross section of production and decay, $\sigma=\sigma_{23} \times \operatorname{BR}\left(\tilde{\chi}_{2}^{0} \rightarrow \tilde{\ell}_{R} \ell\right) \times \operatorname{BR}\left(\tilde{\chi}_{3}^{0} \rightarrow \tilde{\ell}_{R} \ell\right)$, is shown in Fig. 5(d). One can see the combination of the kinematically excluded regions from production and decay. The cross section $\sigma$ reaches up to $65 \mathrm{fb}$. 


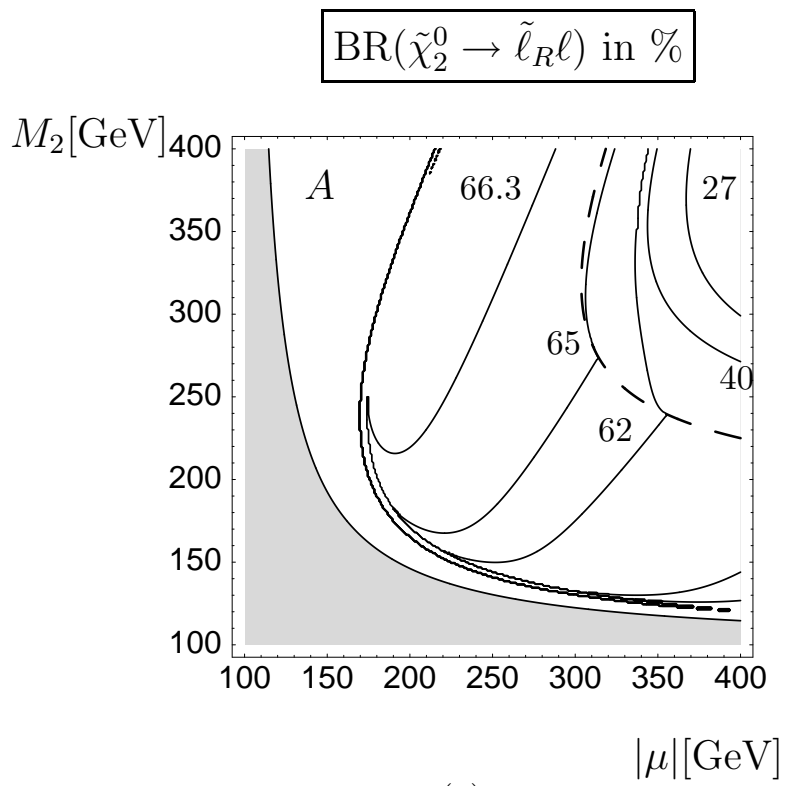

(a)

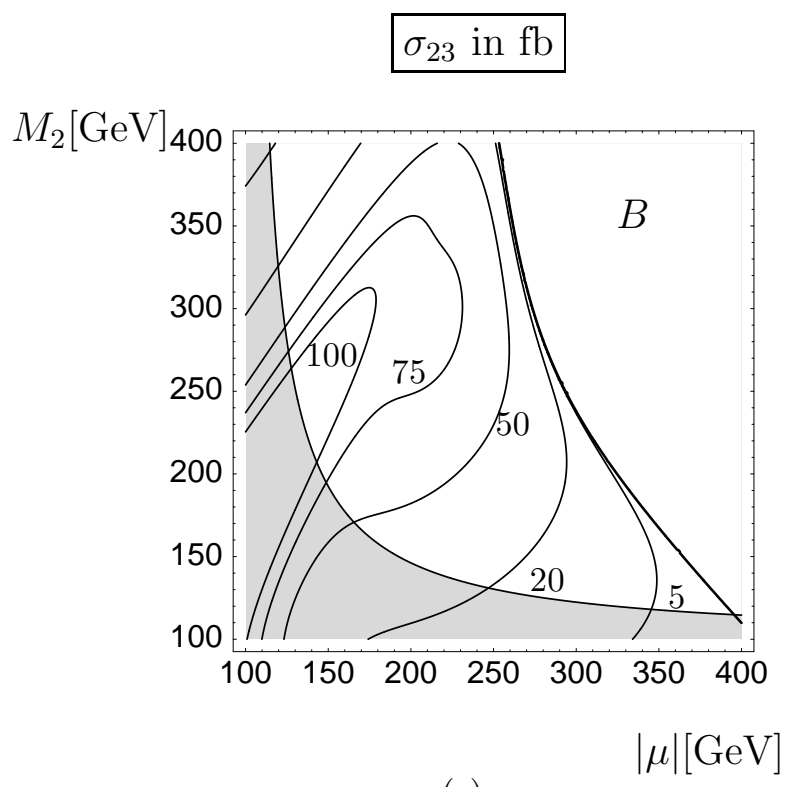

(c)

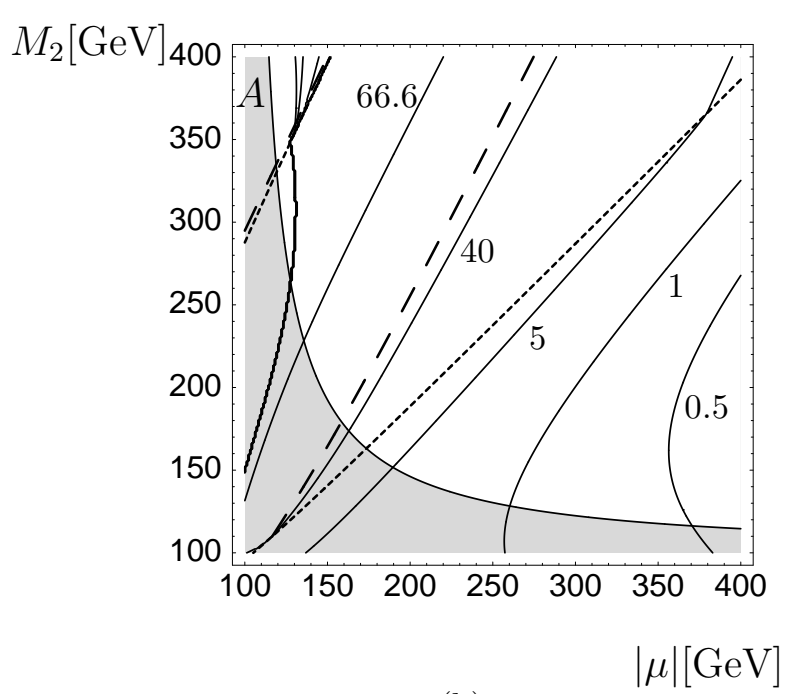

(b)

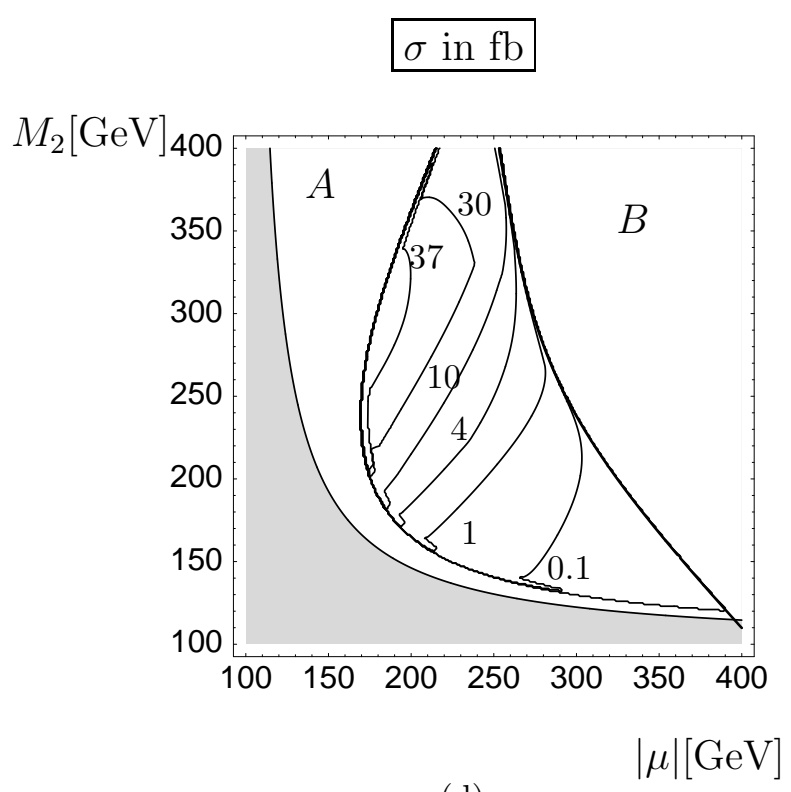

(d)

Figure 5: $|\mu|$ and $M_{2}$ dependence of (a) the neutralino branching ratio $\operatorname{BR}\left(\tilde{\chi}_{2}^{0} \rightarrow \tilde{\ell}_{R} \ell\right)$, (b) the branching ratio $\operatorname{BR}\left(\tilde{\chi}_{3}^{0} \rightarrow \tilde{\ell}_{R} \ell\right)$, (c) the neutralino production cross section $\sigma_{23}=\sigma\left(e^{+} e^{-} \rightarrow \tilde{\chi}_{2}^{0} \tilde{\chi}_{3}^{0}\right)$, and $(\mathrm{d})$ the combined cross section of production and decay, $\sigma=\sigma_{23} \times \operatorname{BR}\left(\tilde{\chi}_{2}^{0} \rightarrow \tilde{\ell}_{R} \ell\right) \times \operatorname{BR}\left(\tilde{\chi}_{3}^{0} \rightarrow \tilde{\ell}_{R} \ell\right)$, for the scenario as defined in Tab. 2. In region $A$ the neutralinos are below the decay threshold, $m_{\chi_{2,3}^{0}}<m_{\tilde{\ell}_{R}}$, and in region $B$ they are above the production threshold, $m_{\chi_{2}^{0}}+m_{\chi_{3}^{0}}>\sqrt{s}=500 \mathrm{GeV}$. In the gray shaded areas the chargino mass is $m_{\chi_{1}^{ \pm}}<100 \mathrm{GeV}$. The dashed contours in (a), (b) indicate the kinematical limit $m_{\chi_{2,3}^{0}}=m_{\chi_{1}^{0}}+m_{Z}$, respectively. The dotted contour in (b) indicates the limit $m_{\chi_{3}^{0}}=m_{W}+m_{\chi_{1}^{\mp}}$. 


\section{$\mathcal{A}_{T}$ in $\%$}

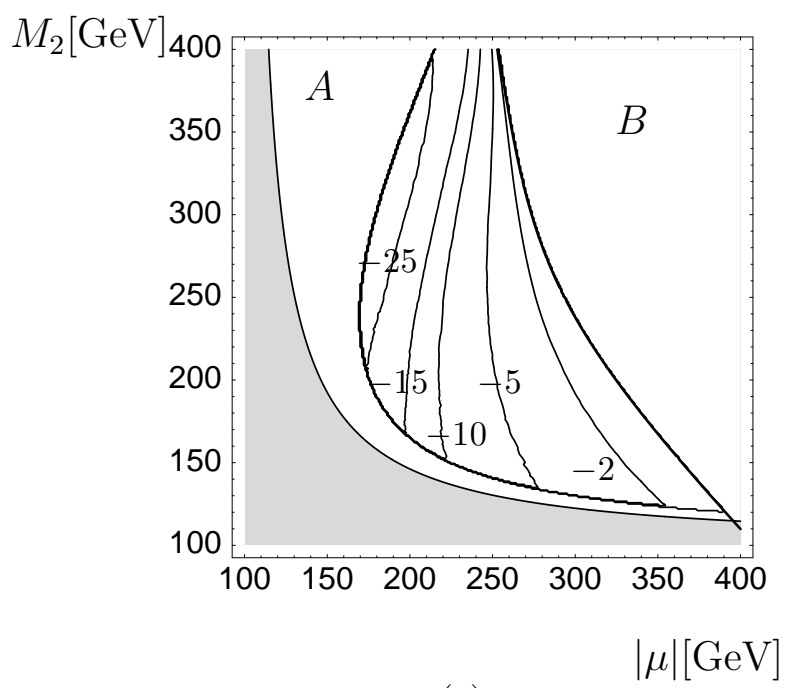

(a)
$S\left[\mathcal{A}_{T}\right]$

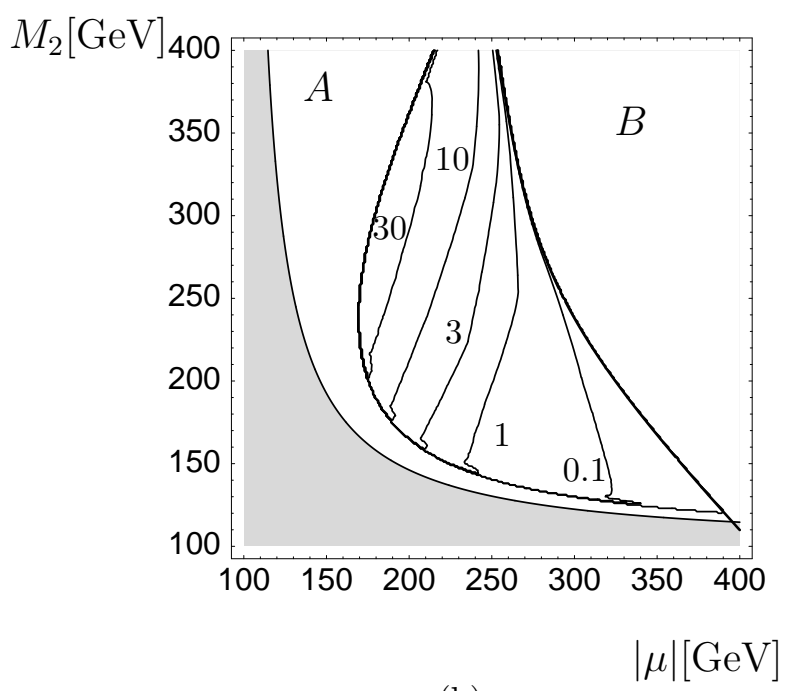

(b)

Figure 6: Contour lines of (a) the asymmetry $\mathcal{A}_{T}$ and (b) its statistical significance $S\left[\mathcal{A}_{T}\right]$ in the $|\mu|-M_{2}$ plane, for the scenario as defined in Tab. 2, In region $A$ neutralino $\tilde{\chi}_{2}^{0}$ is below the decay threshold, $m_{\chi_{2}^{0}}<m_{\tilde{\ell}_{R}}$, and in region $B$ the neutralinos are above the production threshold, $m_{\chi_{2}^{0}}+m_{\chi_{3}^{0}}>\sqrt{s}=500 \mathrm{GeV}$. In the gray shaded areas the chargino mass is $m_{\chi_{1}^{ \pm}}<100 \mathrm{GeV}$.

In Fig. 6, we show the asymmetry $\mathcal{A}_{T}$ and its corresponding significance $S\left[\mathcal{A}_{T}\right]$ in the $|\mu|-M_{2}$ plane. The asymmetry reaches values up to $-30 \%$, while the significance goes up to 50 standard deviations close to the kinematical limit $m_{\chi_{2}^{0}}=m_{\tilde{\ell}_{R}}$, at $M_{2} \approx$ $300 \mathrm{GeV}$ and $|\mu| \approx 180 \mathrm{GeV}$. At that point, the asymmetry of the correlation $\widehat{\mathcal{O}}_{T}^{\prime}$, that does not need the reconstruction of the neutralino momenta, reaches $\hat{\mathcal{A}}_{T}^{\prime}=13 \%$, which corresponds to a significance of about $S\left[\hat{\mathcal{A}}_{T}^{\prime}\right]=25$. 


\section{Summary and conclusions}

We have analyzed CP observables in neutralino production, which are sensitive to the physical phases of the gaugino parameter $M_{1}$, and the higgsino parameter $\mu$. The observables and asymmetries rely on T-odd products in the neutralino spin-spin correlations, which appear on tree-level. The CP-sensitive spin-spin correlations are those terms of the matrix element, which include the polarizations of both neutralinos, with one component normal to the production plane. These spin-spin correlations of the neutralinos can be analyzed via angular distributions of the decay leptons $\tilde{\chi}_{k}^{0} \rightarrow \tilde{\ell} \ell$.

In order to probe the CP-sensitive spin-spin correlation terms, we have defined different T-odd products. One class only involves the final lepton momenta, which has the advantage that it is not necessary to reconstruct the production plane. The second class of T-odd products also includes the neutralino momenta. Based on these T-odd products, we have studied two sorts of CP-sensitive observables. One sort are CP-sensitive observables, which are the expectation values of the T-odd products. The other sort are their corresponding asymmetries, which give the expectation value of the sign of the T-odd products.

In our numerical analysis for $\tilde{\chi}_{2}^{0} \tilde{\chi}_{3}^{0}$ production, we have found that the observables are largest in mixed scenarios with small $\tan \beta$. We have defined theoretical significances to decide, which $\mathrm{CP}$ observable is most sensitive to the $\mathrm{CP}$ phases. For a linear collider with $\sqrt{s}=500 \mathrm{GeV}$ and longitudinally polarized beams, $\left(\mathcal{P}_{-}, \mathcal{P}_{+}\right)=$ $(0.9,-0.6)$, with an integrated luminosity of $\mathcal{L}=500 \mathrm{fb}^{-1}$, the CP-sensitive observables that only include the momenta of the decay leptons yield theoretical significances of $S \lesssim 25$ for $\phi_{1}=0.5 \pi$. We find larger theoretical significances up to $S \lesssim 50$ for the CP-sensitive observables that need a reconstruction of the neutralino momenta. How-

ever, only a detailed experimental study with background and detector simulations can show whether the CP-sensitive observables are accessible. We hope that our results motivate such a study. 


\section{Acknowledgments}

M. T. thanks R. Kögerler for very useful discussions and encouragement. This work is supported by the "Fonds zur Förderung der wissenschaftlichen Forschung" (FWF) of Austria, project No. P18959-N16, and by MICINN project FPA.2006-05294. The authors acknowledge support from EU under the MRTN-CT-2006-035505 and MTRNCT-2006-503369 network proprammes. T. K. is supported by the Portuguese FCT through the projects POCI/FP/81919/2007 and CFTP-FCT UNIT 777, which are partially funded through POCTI (FEDER).

\section{Appendix}

\section{A Decay terms $D$ and $\Sigma_{D}^{c}$}

The coefficients in Eq. (12) of the neutralino decay matrices for the decay into right sleptons $\tilde{\chi}_{k}^{0} \rightarrow \tilde{\ell}_{R}^{-} \ell^{+}$, with $\ell=e, \mu$, are [12]

$$
\begin{aligned}
D_{k} & =\frac{g^{2}}{2}\left|f_{\ell k}^{R}\right|^{2}\left(m_{\chi_{k}^{0}}^{2}-m_{\tilde{\ell}_{R}}^{2}\right), \\
\Sigma_{D_{k}}^{c} & ={ }_{(-)}^{+} g^{2}\left|f_{\ell k}^{R}\right|^{2} m_{\chi_{k}^{0}}\left(s_{\chi_{k}}^{c} \cdot p_{\ell}\right),
\end{aligned}
$$

where the sign in parenthesis holds for the charge conjugated process $\tilde{\chi}_{k}^{0} \rightarrow \tilde{\ell}_{R}^{+} \ell^{-}$. For the decay into the left sleptons $\tilde{\chi}_{k}^{0} \rightarrow \tilde{\ell}_{L}^{-} \ell^{+}, \ell=e, \mu$, the coefficients are

$$
\begin{aligned}
D_{k} & =\frac{g^{2}}{2}\left|f_{\ell k}^{L}\right|^{2}\left(m_{\chi_{k}^{0}}^{2}-m_{\tilde{\ell}_{L}}^{2}\right), \\
\Sigma_{D_{k}}^{c} & ={ }_{(+)} g^{2}\left|f_{\ell k}^{L}\right|^{2} m_{\chi_{k}^{0}}\left(s_{\chi_{k}}^{c} \cdot p_{\ell}\right),
\end{aligned}
$$

where the sign in parenthesis holds for the charge conjugated process $\tilde{\chi}_{k}^{0} \rightarrow \tilde{\ell}_{L}^{+} \ell^{-}$.

In order to reduce the free MSSM parameters, we parametrize the slepton masses with an approximate solution to the renormalization group equations (RGE) [23]

$$
\begin{aligned}
& m_{\tilde{\ell}_{R}}^{2}=m_{0}^{2}+m_{\ell}^{2}+0.23 M_{2}^{2}-m_{Z}^{2} \cos 2 \beta \sin ^{2} \theta_{W}, \\
& m_{\tilde{\ell}_{L}}^{2}=m_{0}^{2}+m_{\ell}^{2}+0.79 M_{2}^{2}+m_{Z}^{2} \cos 2 \beta\left(-\frac{1}{2}+\sin ^{2} \theta_{W}\right),
\end{aligned}
$$




$$
m_{\tilde{\nu}_{\ell}}^{2}=m_{0}^{2}+m_{\ell}^{2}+0.79 M_{2}^{2}+\frac{1}{2} m_{Z}^{2} \cos 2 \beta
$$

with $m_{0}$ the common scalar mass parameter at the GUT scale.

\section{B Momentum and polarization vectors}

We choose a coordinate system with the $z$-axis along the $\vec{p}_{e^{-}}$direction in the centerof-mass system. The four-momenta of the neutralinos $\tilde{\chi}_{i}^{0}$ and $\tilde{\chi}_{j}^{0}$ are

$$
p_{\chi_{i, j}}=q\left(\frac{E_{\chi_{i, j}}}{q}, \mp \sin \theta, 0, \mp \cos \theta\right)
$$

with their energies and common momentum

$$
E_{\chi_{i, j}}=\frac{s+m_{\chi_{i, j}}^{2}-m_{\chi_{j, i}}^{2}}{2 \sqrt{s}}, \quad q=\frac{\lambda^{\frac{1}{2}}\left(s, m_{\chi_{i}}^{2}, m_{\chi_{j}}^{2}\right)}{2 \sqrt{s}}
$$

respectively, and the kinematic function $\lambda(a, b, c)=a^{2}+b^{2}+c^{2}-2(a b+a c+b c)$. The scattering angle is $\theta \Varangle\left(\vec{p}_{e^{-}}, \vec{p}_{\chi_{j}}\right)$, whereas the azimuthal angle can be set to zero, due to rotational invariance around the beam axis [24].

The three spin basis vectors of $\tilde{\chi}_{i}^{0}$ and $\tilde{\chi}_{j}^{0}$ are chosen to be

$$
\begin{aligned}
& s_{\chi_{i, j}}^{1}=\left(0, \frac{\vec{s}_{\chi_{i, j}}^{2} \times \vec{s}_{\chi_{i, j}}^{3}}{\left|\vec{s}_{\chi_{i, j}}^{2} \times \vec{s}_{\chi_{i, j}}^{3}\right|}\right)= \pm(0, \cos \theta, 0,-\sin \theta), \\
& s_{\chi_{i, j}}^{2}=\left(0, \frac{\vec{p}_{e^{-}} \times \vec{p}_{\chi_{i, j}}}{\left|\vec{p}_{e^{-}} \times \vec{p}_{\chi_{i, j}}\right|}\right)=(0,0,1,0), \\
& s_{\chi_{i, j}}^{3}=\frac{1}{m_{\chi_{i, j}}}\left(q, \frac{E_{\chi_{i, j}}}{q} \vec{p}_{\chi_{i, j}}\right)=\frac{E_{\chi_{i, j}}}{m_{\chi_{i, j}}}\left(\frac{q}{E_{\chi_{i, j}}}, \mp \sin \theta, 0, \mp \cos \theta\right) .
\end{aligned}
$$

They fulfill the orthonormality relations $s_{\chi_{k}}^{c} \cdot s_{\chi_{k}}^{d}=-\delta^{c d}, s_{\chi_{k}}^{c} \cdot p_{\chi_{k}}=0$, and the completeness relation $[6,18]$

$$
\sum_{c} s_{\chi_{k}}^{c, \mu} \cdot s_{\chi_{k}}^{c, \nu}=-g^{\mu \nu}+\frac{p_{\chi_{k}}^{\mu} p_{\chi_{k}}^{\nu}}{m_{\chi_{k}}^{2}}
$$

The four-momenta of the leptons in the decays $\tilde{\chi}_{i}^{0} \rightarrow \tilde{\ell} \ell$, and $\tilde{\chi}_{j}^{0} \rightarrow \tilde{\ell}^{\prime} \ell^{\prime}$, are

$$
p_{\ell}=\left|\vec{p}_{\ell}\right|\left(1, \cos \phi_{\ell} \sin \theta_{\ell}, \sin \phi_{\ell} \sin \theta_{\ell}, \cos \theta_{\ell}\right),
$$




$$
p_{\ell^{\prime}}=\left|\vec{p}_{\ell^{\prime}}\right|\left(1, \cos \phi_{\ell^{\prime}} \sin \theta_{\ell^{\prime}}, \sin \phi_{\ell^{\prime}} \sin \theta_{\ell^{\prime}}, \cos \theta_{\ell^{\prime}}\right),
$$

respectively, with

$$
\left|\vec{p}_{\ell}\right|=\frac{m_{\chi_{i}}^{2}-m_{\tilde{\ell}}^{2}}{2\left(E_{\chi_{i}}+q \cos \vartheta_{\ell}\right)}, \quad\left|\vec{p}_{\ell^{\prime}}\right|=\frac{m_{\chi_{j}}^{2}-m_{\tilde{\ell}^{\prime}}^{2}}{2\left(E_{\chi_{j}}-q \cos \vartheta_{\ell^{\prime}}\right)},
$$

and the decay angles

$$
\begin{aligned}
\cos \vartheta_{\ell} & =\sin \theta \sin \theta_{\ell} \cos \phi_{\ell}+\cos \theta \cos \theta_{\ell}, \\
\cos \vartheta_{\ell^{\prime}} & =\sin \theta \sin \theta_{\ell^{\prime}} \cos \phi_{\ell^{\prime}}+\cos \theta \cos \theta_{\ell^{\prime}} .
\end{aligned}
$$

With these definitions, the T-odd products $f^{a b}$ (19) of the spin-spin correlation terms in the laboratory system are

$$
\begin{aligned}
f^{12} & =-\frac{1}{2} E_{\chi_{i}} s q \sin ^{2} \theta, & f^{21} & =\frac{1}{2} E_{\chi_{j}} s q \sin ^{2} \theta, \\
f^{23} & =\frac{1}{4} m_{\chi_{j}} s q \sin (2 \theta), & f^{32} & =-\frac{1}{4} m_{\chi_{i}} s q \sin (2 \theta) .
\end{aligned}
$$

\section{Phase space}

The Lorentz invariant phase space element in Eq. (11) is given by $[12,24]$

$$
\mathrm{d} \operatorname{Lips}=\frac{1}{(2 \pi)^{2}} \mathrm{~d} \operatorname{Lips}\left(s, p_{\chi_{i}}, p_{\chi_{j}}\right) \mathrm{d} s_{\chi_{i}} \mathrm{dLips}\left(s_{\chi_{i}}, p_{\tilde{\ell}}, p_{\ell}\right) \mathrm{d} s_{\chi_{j}} \mathrm{dLips}\left(s_{\chi_{j}}, p_{\tilde{\ell}^{\prime}}, p_{\ell^{\prime}}\right),
$$

with $s_{\chi_{i, j}}=p_{\chi_{i, j}}^{2}$. The different factors of the phase space element are

$$
\begin{aligned}
& \mathrm{d} \operatorname{Lips}\left(s, p_{\chi_{i}}, p_{\chi_{j}}\right)=\frac{1}{8 \pi} \frac{q}{\sqrt{s}} \sin \theta \mathrm{d} \theta, \\
& \mathrm{d} \operatorname{Lips}\left(s_{\chi_{i}}, p_{\tilde{\ell}}, p_{\ell}\right)=\frac{1}{2(2 \pi)^{2}} \frac{\left|\vec{p}_{\ell}\right|^{2}}{m_{\chi_{i}}^{2}-m_{\tilde{\ell}}^{2}} \sin \theta_{\ell} \mathrm{d} \theta_{\ell} \mathrm{d} \phi_{\ell}, \\
& \mathrm{d} \operatorname{Lips}\left(s_{\chi_{j}}, p_{\tilde{\ell}^{\prime}}, p_{\ell^{\prime}}\right)=\frac{1}{2(2 \pi)^{2}} \frac{\left|\vec{p}_{\ell^{\prime}}\right|^{2}}{m_{\chi_{j}}^{2}-m_{\tilde{\ell}^{\prime}}^{2}} \sin \theta_{\ell^{\prime}} \mathrm{d} \theta_{\ell^{\prime}} \mathrm{d} \phi_{\ell^{\prime}} .
\end{aligned}
$$


We use the narrow width approximation for the propagators in Eq. (12),

$$
\int\left|\Delta\left(\tilde{\chi}_{i, j}^{0}\right)\right|^{2} \mathrm{~d} s_{\chi_{i, j}}=\frac{\pi}{m_{\chi_{i, j}} \Gamma_{\chi_{i, j}}}
$$

which is justified for $\Gamma / m \ll 1$, which holds in our case with $\Gamma \lesssim \mathcal{O}(1 \mathrm{GeV})$. Note, however, that the naive $\mathcal{O}(\Gamma / m)$-expectation of the error can easily receive large offshell corrections of an order of magnitude and more, in particular at threshold, or due to interferences with other resonant or non-resonant processes. For recent discussions of these issues, see Ref. [25].

\section{References}

[1] F. Csikor, Z. Fodor and J. Heitger, Phys. Rev. Lett. 82 (1999) 21 arXiv:hep-ph/9809291.

[2] A. Riotto, arXiv:hep-ph/9807454; W. Bernreuther, Lect. Notes Phys. 591 (2002) 237 arXiv:hep-ph/0205279.

[3] J. R. Ellis, J. S. Lee and A. Pilaftsis, JHEP 0810 (2008) 049 arXiv:0808.1819 [hep-ph]].

[4] T. Ibrahim and P. Nath, Phys. Rev. D 57 (1998) 478 [Erratum-ibid. D 58 (1998) 019901, D 60 (1999) 079903, D 60 (1999) 119901] arXiv:hep-ph/9708456;

M. Brhlik, G. J. Good and G. L. Kane, Phys. Rev. D 59, 115004 (1999) arXiv:hep-ph/9810457;

A. Bartl, T. Gajdosik, W. Porod, P. Stockinger and H. Stremnitzer, Phys. Rev. D 60 (1999) 073003 arXiv:hep-ph/9903402;

V. D. Barger, T. Falk, T. Han, J. Jiang, T. Li and T. Plehn, Phys. Rev. D 64, 056007 (2001) arXiv:hep-ph/0101106;

S. Yaser Ayazi and Y. Farzan, Phys. Rev. D $74 \quad$ (2006) 055008 arXiv:hep-ph/0605272.

[5] J. Brau et al. [ILC Collaboration], arXiv:0712.1950 [physics.acc-ph];

J. A. Aguilar-Saavedra et al. [ECFA/DESY LC Physics Working Group], arXiv:hep-ph/0106315;

T. Abe et al. [American Linear Collider Working Group], arXiv:hep-ex/0106055; 
K. Abe et al. [ACFA Linear Collider Working Group], arXiv:hep-ph/0109166;

J. A. Aguilar-Saavedra et al., Eur. Phys. J. C 46 (2006) 43 arXiv:hep-ph/0511344.

[6] G. A. Moortgat-Pick, H. Fraas, A. Bartl and W. Majerotto, Eur. Phys. J. C 9 (1999) 521 [Erratum-ibid. C 9 (1999) 549] arXiv:hep-ph/9903220];

G. A. Moortgat-Pick, Doctoral thesis "Spin effects in chargino/neutralino production and decay" (in German), Universität Würzburg (1999).

[7] J. L. Kneur and G. Moultaka, Phys. Rev. D 61 (2000) 095003 arXiv:hep-ph/9907360;

V. D. Barger, T. Han, T. J. Li and T. Plehn, Phys. Lett. B 475 (2000) 342 arXiv:hep-ph/9907425;

S. Y. Choi, H. S. Song and W. Y. Song, Phys. Rev. D 61 (2000) 075004 arXiv:hep-ph/9907474;

S. Y. Choi, J. Kalinowski, G. A. Moortgat-Pick and P. M. Zerwas, Eur. Phys. J. C 22 (2001) 563 [Addendum-ibid. C 23 (2002) 769] arXiv:hep-ph/0108117];

G. J. Gounaris and C. Le Mouel, Phys. Rev. D 66 (2002) 055007 arXiv:hep-ph/0204152;

S. Y. Choi, Phys. Rev. D 69 (2004) 096003 arXiv:hep-ph/0308060.

[8] G. Valencia, arXiv:hep-ph/9411441, and references therein;

G.C. Branco, L. Lavoura, and J.P. Silva, CP violation, Oxford University Press, New York, 1999.

[9] H. K. Dreiner, O. Kittel and F. von der Pahlen, JHEP 0801, 017 (2008) arXiv:0711.2253 [hep-ph]].

[10] A. Bartl, K. Hohenwarter-Sodek, T. Kernreiter, O. Kittel and M. Terwort, Nucl. Phys. B 802 (2008) 77 [arXiv:0802.3592 [hep-ph]].

[11] Y. Kizukuri and N. Oshimo, Phys. Lett. B 249 (1990) 449;

A. Bartl, H. Fraas, O. Kittel and W. Majerotto, Phys. Rev. D 69 (2004) 035007 arXiv:hep-ph/0308141; Eur. Phys. J. C 36 (2004) 233 arXiv:hep-ph/0402016;

A. Bartl, T. Kernreiter and O. Kittel, Phys. Lett. B 578, 341 (2004) arXiv:hep-ph/0309340;

S. Y. Choi, M. Drees, B. Gaissmaier and J. Song, Phys. Rev. D 69, 035008 
(2004) arXiv:hep-ph/0310284;

S. Y. Choi and Y. G. Kim, Phys. Rev. D 69, 015011 (2004) arXiv:hep-ph/0311037;

J. A. Aguilar-Saavedra, Nucl. Phys. B 697 (2004) 207 arXiv:hep-ph/0404104;

S. Y. Choi, M. Drees and B. Gaissmaier, Phys. Rev. D 70 (2004) 014010 arXiv:hep-ph/0403054;

A. Bartl, H. Fraas, S. Hesselbach, K. Hohenwarter-Sodek and G. A. MoortgatPick, JHEP 0408 (2004) 038 arXiv:hep-ph/0406190;

S. Y. Choi, B. C. Chung, J. Kalinowski, Y. G. Kim and K. Rolbiecki, Eur. Phys. J. C 46, 511 (2006) arXiv:hep-ph/0504122.

[12] O. Kittel, arXiv:hep-ph/0504183.

[13] A. Bartl, H. Fraas, S. Hesselbach, K. Hohenwarter-Sodek, T. Kernreiter and G. A. Moortgat-Pick, JHEP 0601, 170 (2006) arXiv:hep-ph/0510029].

[14] G. A. Moortgat-Pick et al., Phys. Rept. 460, 131 (2008) arXiv:hep-ph/0507011; S. Y. Choi, M. Drees and J. Song, JHEP 0609, 064 (2006) arXiv:hep-ph/0602131;

A. Bartl, K. Hohenwarter-Sodek, T. Kernreiter and O. Kittel, JHEP 0709, 079 (2007) arXiv:0706.3822 [hep-ph]].

[15] A. Bartl, H. Fraas, T. Kernreiter and O. Kittel, Eur. Phys. J. C 33, 433 (2004) arXiv:hep-ph/0306304;

J. A. Aguilar-Saavedra, Phys. Lett. B 596, 247 (2004) arXiv:hep-ph/0403243;

P. Langacker, G. Paz, L. T. Wang and I. Yavin, JHEP 0707, 055 (2007) arXiv:hep-ph/0702068;

J. Ellis, F. Moortgat, G. Moortgat-Pick, J. M. Smillie and J. Tattersall, arXiv:0809.1607 [hep-ph].

[16] A. Bartl, H. Fraas and W. Majerotto, Nucl. Phys. B 278 (1986) 1.

[17] H. E. Haber and G. L. Kane, Phys. Rept. 117 (1985) 75.

[18] H. E. Haber, Proceedings of the 21st SLAC Summer Institute on Particle Physics, eds. L. DeProcel, Ch. Dunwoodie, Stanford 1993, 231, arXiv:hep-ph/9405376.

[19] D. Atwood and A. Soni, Phys. Rev. D 45, 2405 (1992);

M. Diehl and O. Nachtmann, Z. Phys. C 62, 397 (1994); 
B. Grzadkowski and J. F. Gunion, Phys. Lett. B 350, 218 (1995) arXiv:hep-ph/9501339.

[20] A. Bartl, H. Fraas, K. Hohenwarter-Sodek, T. Kernreiter, G. Moortgat-Pick and A. Wagner, Phys. Lett. B 644, 165 (2007) arXiv:hep-ph/0610431.

[21] Y. Kizukuri and N. Oshimo, arXiv:hep-ph/9310224;

S. Y. Choi, A. Djouadi, M. Guchait, J. Kalinowski, H. S. Song and P. M. Zerwas, Eur. Phys. J. C 14, 535 (2000) arXiv:hep-ph/0002033;

A. Bartl, H. Fraas, O. Kittel and W. Majerotto, Phys. Lett. B 598, 76 (2004) arXiv:hep-ph/0406309;

O. Kittel, A. Bartl, H. Fraas and W. Majerotto, Phys. Rev. D 70, 115005 (2004) arXiv:hep-ph/0410054;

J. A. Aguilar-Saavedra, Nucl. Phys. B 717, 119 (2005) arXiv:hep-ph/0410068;

A. Bartl, H. Fraas, S. Hesselbach, K. Hohenwarter-Sodek, T. Kernreiter and G. Moortgat-Pick, Eur. Phys. J. C 51, 149 (2007) arXiv:hep-ph/0608065;

A. Bartl, K. Hohenwarter-Sodek, T. Kernreiter and H. Rud, Eur. Phys. J. C 36 (2004) 515 arXiv:hep-ph/0403265.

[22] C. Amsler et al. [Particle Data Group], Phys. Lett. B 667 (2008) 1.

[23] L. E. Ibanez and C. Lopez, Nucl. Phys. B 233 (1984) 511;

L. E. Ibanez, C. Lopez and C. Munoz, Nucl. Phys. B 256 (1985) 218;

L. J. Hall and J. Polchinski, Phys. Lett. B 152 (1985) 335.

[24] E. Byckling, K. Kajantie, Particle Kinematics, John Wiley \& Sons, 1973.

[25] K. Hagiwara et al., Phys. Rev. D 73 (2006) 055005 arXiv:hep-ph/0512260;

D. Berdine, N. Kauer and D. Rainwater, Phys. Rev. Lett. 99, 111601 (2007) arXiv:hep-ph/0703058;

N. Kauer, Phys. Lett. B 649, 413 (2007) arXiv:hep-ph/0703077); JHEP 0804, 055 (2008) arXiv:0708.1161 [hep-ph]];

C. F. Uhlemann and N. Kauer, Nucl. Phys. B 814, 195 (2009) arXiv:0807.4112 [hep-ph]];

M. A. Gigg and P. Richardson, arXiv:0805.3037 [hep-ph]. 